\title{
Ions Generated from Uranyl Nitrate Solutions by Electrospray Ionization (ESI) and Detected with Fourier Transform Ion-Cyclotron Resonance (FT-ICR) Mass Spectrometry
}

\author{
Sofie Pasilis, * Árpád Somogyi, Kristin Herrmann, \\ and Jeanne E. Pemberton \\ Department of Chemistry, University of Arizona, Tucson, Arizona, USA
}

\begin{abstract}
Electrospray ionization (ESI) of uranyl nitrate solutions generates a wide variety of positively and negatively charged ions, including complex adducts of uranyl ions with methoxy, hydroxy, and nitrate ligands. In the positive ion mode, ions detected by Fourier transform ion cyclotron resonance (FT-ICR) mass spectrometry are sensitive to instrumental tuning parameters such as quadrupole operating frequency and trapping time. Positive ions correspond to oligomeric uranyl nitrate species that can be characterized as having a general formula of $\left[\left(\mathrm{UO}_{2}\right)_{\mathrm{n}}(\mathrm{A})_{\mathrm{m}}\left(\mathrm{CH}_{3} \mathrm{OH}\right)_{\mathrm{s}}\right]^{+}$or $\left[\left(\mathrm{UO}_{2}\right)_{\mathrm{n}}(\mathrm{O})(\mathrm{A})_{\mathrm{m}}\left(\mathrm{CH}_{3} \mathrm{OH}\right)_{\mathrm{s}}\right]^{+}$with $n=1-4, \mathrm{~m}=1-7, \mathrm{~s}=0$ or 1 , and $\mathrm{A}=\mathrm{OH}, \mathrm{NO}_{3}, \mathrm{CH}_{3} \mathrm{O}$ or a combination of these, although the formation of $\mathrm{NO}_{3}$-containing species is preferred. In the negative ion mode, complexes of the form $\left[\left(\mathrm{UO}_{2}\right)\left(\mathrm{NO}_{3}\right)_{\mathrm{m}}\right]^{-}(\mathrm{m}=$ 1-3) are detected, although the formation of the oxo-containing ions $\left[\left(\mathrm{UO}_{2}\right)(\mathrm{O})_{\mathrm{n}}\left(\mathrm{NO}_{3}\right)_{\mathrm{m}}\right]^{-}(n=$ $1-2, \mathrm{~m}=1-2)$ and the hydroxy-containing ions $\left[\left(\mathrm{UO}_{2}\right)(\mathrm{OH})_{\mathrm{n}}\left(\mathrm{NO}_{3}\right)_{\mathrm{m}}\right]^{-}(n=1-2, \mathrm{~m}=0-1)$ are also observed. The extent of coordinative unsaturation of both positive and negative ions can be determined by ligand association/exchange and H/D exchange experiments using $\mathrm{D}_{2} \mathrm{O}$ and $\mathrm{CD}_{3} \mathrm{OD}$ as neutral reaction partners in the gas-phase. Positive ions are of varying stability and reactivity and may fragment extensively upon collision with $\mathrm{D}_{2} \mathrm{O}, \mathrm{CD}_{3} \mathrm{OD}$ and $\mathrm{N}_{2}$ in sustained off-resonance irradiation/collision-induced dissociation (SORI-CID) experiments. Electron-transfer reactions, presumably occurring during electrospray ionization but also in SORI-CID, can result in reduction of $\mathrm{U}(\mathrm{VI})$ to $\mathrm{U}(\mathrm{V})$ and perhaps even $\mathrm{U}(\mathrm{IV})$. (J Am Soc Mass Spectrom 2006, 17, 230-240) (c) 2006 American Society for Mass Spectrometry
\end{abstract}

$\mathrm{T}$ The aqueous chemistry of uranium influences important technological, biological, and geochemical processes involving this element, including its mobility and fate in the geologic subsurface, bioavailability and carcinogenicity, the genesis of uranium mineral deposits and uranium mining, nuclear fuel reprocessing, and the migration of nuclear wastes. However, while great strides have been made in understanding uranium speciation in aqueous systems and the thermodynamic properties of organic and inorganic uranium complexes, the complexity of uranium chemistry sustains existing confusion regarding the existence of individual species under particular conditions of $\mathrm{pH}$, temperature, ionic strength, solution concentration of uranium, and potential complexing ligands. One major difficulty is the paucity of methods that can be used to distinguish specific molecular species at low concentra-

Published online January 18, 2006

Address reprint requests to Dr. J. E. Pemberton, Department of Chemistry, University of Arizona, 1306 East University Blvd., Tucson, AZ 85721, USA.E-mail: pembertn@u.arizona.edu

* Current address: Chemical Sciences Division, Oak Ridge National Laboratory, Oak Ridge, TN 37831. tion in solution. Electrospray ionization mass spectrometry (ESI-MS) can provide molecular weight and structural information and has been used successfully for molecular characterization of $\mathrm{U}(\mathrm{VI})$ complexes with ligands of environmental or biological interest [1-5]. However, much fundamental work on the complex gas-phase chemistry of $\mathrm{U}(\mathrm{VI})$ and $\mathrm{U}(\mathrm{VI})$-containing species remains to be done before ESI-MS can be used to characterize U(VI) species in natural waters.

Previous studies on the gas-phase reactivity of uranium ions have been reported. Many of these studies have focused on the reactions of $\mathrm{U}^{+}$and $\mathrm{UO}^{+}$with alkanes [6, 7], alkenes [8], alcohols, thiols and ethers [9], arenes [10], and other small molecules [11-16]. Recently, the hydration reactivities of $\mathrm{UO}^{+}, \mathrm{UO}_{2}^{+}$, and $\mathrm{UO}_{2}^{2+}$ were examined using secondary ion mass spectrometry [17]. These studies used surface ionization [11, 12], pulsed glow discharge [13, 14], or laser ablation $[6-11,13,16,17]$ techniques to create uranium ions for study. In addition, several studies have used electrospray ionization combined with an ion trap instrument to generate various $\mathrm{UO}_{2}^{2+}$-ligand complexes from solu- 
tion and study their gas-phase reactivity with neutral solvent molecules [18-21].

In the present study, we use Fourier transform ion cyclotron resonance (FT-ICR) mass spectrometry to elucidate the gas-phase behavior of uranyl-containing species. To the best of our knowledge, this is the first systematic investigation using FT-ICR mass spectrometry to obtain accurate mass measurements on gasphase uranyl-containing ions, as well as to examine ligand association/exchange and H/D exchange reactions of these species.

\section{Experimental}

Uranyl nitrate hexahydrate, $\mathrm{UO}_{2}\left(\mathrm{NO}_{3}\right)_{2} \cdot 6 \mathrm{H}_{2} \mathrm{O}(\mathrm{J}$. T. Baker) was received as a gift from the University of Arizona Radiation Control Office and recrystallized before use. Water $(18 \mathrm{M} \Omega$, total organic carbon $<10$ $\mathrm{ppb}$ ) was purified with a Milli-Q UV system (Millipore Corporation, Billerica, $\mathrm{MA}) . \mathrm{D}_{2} \mathrm{O}(99.9 \% \mathrm{D})$ and $\mathrm{CD}_{3} \mathrm{OD}$ $(99.8 \% \mathrm{D})$ were purchased from Cambridge Isotopes (Andover, MA) and were introduced into the ICR cell through a leak valve after several steps of degassing.

Samples were prepared from a $1 \mathrm{mM}$ stock solution of uranyl nitrate in $\mathrm{H}_{2} \mathrm{O}$. Samples for FT-ICR MS measurements were prepared by diluting the stock solution with methanol. Solutions from which positive ion spectra were acquired were $330 \mu \mathrm{M}$ in $\mathrm{UO}_{2}^{2+}$ and $660 \mu \mathrm{M}$ in $\mathrm{NO}_{3}^{-}$in a $66 \% \mathrm{CH}_{3} \mathrm{OH} / 33 \% \mathrm{H}_{2} \mathrm{O}(\mathrm{vol} / \mathrm{vol})$ solvent system; solutions from which negative ion spectra were acquired were $50 \mu \mathrm{M}$ in $\mathrm{UO}_{2}^{2+}$ and $100 \mu \mathrm{M}$ in $\mathrm{NO}_{3}^{-}$in a $95 \% \mathrm{CH}_{3} \mathrm{OH} / 5 \% \mathrm{H}_{2} \mathrm{O}$ (vol/vol) solvent system.

An IonSpec $4.7 \mathrm{~T}$ Fourier ion cyclotron resonance (FT-ICR) instrument (Lake Forest, CA) was used for ultrahigh resolution/accurate mass measurements, as well as for gas-phase ligand association/exchange and $\mathrm{H} / \mathrm{D}$ exchange studies. Ions were generated using an Analytica (Branford, CT) second-generation electrospray source. Ions were introduced into the instrument by infusing sample solutions using a stainless steel microelectrospray needle $(0.10 \mathrm{~mm}$ i.d. $)$ at a flow rate of $2 \mu \mathrm{L} / \mathrm{min}$. The capillary temperature was kept at $60^{\circ} \mathrm{C}$. For positively charged ions, a needle voltage of $3.8 \mathrm{kV}$ was applied, while $-2.5 \mathrm{kV}$ was used in the negative ion mode. Once generated, the ions pass through a skimmer that retards the passage of neutral molecules and restricts the pressure, and then are accumulated in an external RF-only hexapole for $400-1000 \mathrm{~ms}$. The accumulated ions are passed through the high-speed shutter into the RF-only quadrupole ion guide where they are focused and guided into the ICR cell where they are trapped. The quadrupole ion guide acts as a mass filter and has two operating frequencies, 1820 and $965 \mathrm{kHz}$. According to the manufacturer, these two frequencies allow trapping of ions in the ranges of $\mathrm{m} / \mathrm{z}$ $100-1000$ and $\mathrm{m} / \mathrm{z} 400-2500$, respectively.

This instrument was modified in-house to accommodate ligand association/exchange and H/D exchange reactions by incorporating the pulsed-leak configuration described by Jiao et al. [22]. Typical analyzer base pressures are $7 \times 10^{-11}$ torr. The pulsed-leak configuration allows introduction of reagent gas into the analyzer region at a constant partial pressure for a specified period of time.

The ion gauge used to determine reagent gas pressures was calibrated using betaine, which contains one exchangeable proton with a known exchange rate constant [23]. The partial pressures of $\mathrm{D}_{2} \mathrm{O}$ and $\mathrm{CD}_{3} \mathrm{OD}$ exchange reagents were corrected for ion gauge sensitivities [24]. The corrected, calibrated pressure of $\mathrm{D}_{2} \mathrm{O}$ gas used for these experiments was $(2.4 \pm 0.5) \times 10^{-8}$ torr, while the corrected, calibrated pressure of $\mathrm{CD}_{3} \mathrm{OD}$ gas was $(4.8 \pm 0.3) \times 10^{-8}$ torr. Ion-molecule reaction times were varied from 10 to $180 \mathrm{~s}$. For H/D and ligand exchange experiments, all trapped ions except those of interest are ejected from the ICR cell via a chirp frequency sweep. The isolated ions are allowed to cool for several seconds before the reagent gas is leaked into the analyzer region. At the end of a specified reaction time under constant exchange reagent pressure, neutral reagent gas was pumped away during a $30 \mathrm{~s}$ pump-down to reach the very low pressures necessary for FT-ICR analysis. Pressure in the ICR cell at the time of detection was $\sim 3.5 \times 10^{-9}$ torr.

Various spectral calibration procedures were used to obtain exact mass values. For positively-charged uranyl nitrate-containing species of $m / z>900$, a peak matching procedure using Ultramark 1621 (Lancaster Synthesis, Inc., Lancashire, UK) was used. For positive species of lower mass, ions previously reported were used for selfinternal calibration. For MS/MS and $\mathrm{D}_{2} \mathrm{O} / \mathrm{CD}_{3} \mathrm{OD}$ ionmolecule reaction spectra, ions previously assigned using Ultramark 1621 were used whenever possible for selfinternal calibration. For negative ions, linear alkylbenzene sulfonates (LAS) were initially used for internal calibration and to verify ion assignments. For subsequent studies, unambiguously-assigned uranyl-containing ions were used for self-internal calibration. Ions used for calibration are marked with an asterisk in the tables.

\section{Results and Discussion}

\section{Influence of Tuning Parameters on ESI Mass Spectra}

Electrospray ionization of uranyl nitrate solutions generates a wide variety of positively charged uranyl-containing ions. These species include not only the relatively simple $\left[\left(\mathrm{UO}_{2}\right)(\mathrm{A}) \mathrm{S}_{\mathrm{n}}\right]^{+}$ions, where $\mathrm{A}$ is $\mathrm{CH}_{3} \mathrm{O}, \mathrm{OH}$, or $\mathrm{NO}_{3}$ and $\mathrm{S}$ is either $\mathrm{CH}_{3} \mathrm{OH}$ or $\mathrm{H}_{2} \mathrm{O}$ as described in Van Stipdonk et al. [18, 20, 21] and in Chien et al. [19], but also more complex positively charged oligomeric species at higher $m / z$, as well as a number of simple, nitrate-containing, negatively-charged species.

In the positive ion mode, the types of uranyl-containing ions detected using FT-ICR are dependent on such 


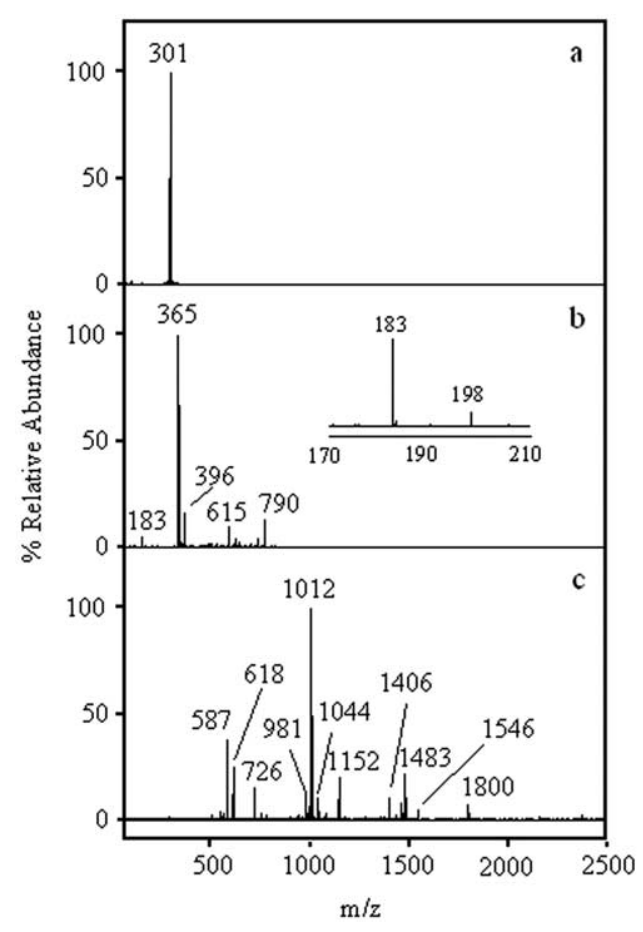

Figure 1. Ions resulting from a solution $330 \mu \mathrm{M}$ in $\mathrm{UO}_{2}^{2+}$ and 660 $\mu \mathrm{M}$ in $\mathrm{NO}_{3}^{-}$in a $66 \% \mathrm{CH}_{3} \mathrm{OH} / 33 \% \mathrm{H}_{2} \mathrm{O}$ (vol/vol) solvent system to indicate the influence of quadrupole trapping frequency, pulse length, and hexapole trapping time on ions in positive ion mass spectra. (a) $1820 \mathrm{kHz}, 2.881 \mathrm{~ms}, 1000 \mathrm{~ms}$, (b) $965 \mathrm{kHz}, 3.167 \mathrm{~ms}, 400$ ms, (c) $965 \mathrm{kHz}, 3.800 \mathrm{~ms}, 1000 \mathrm{~ms}$.

instrumental tuning parameters as hexapole trapping time, quadrupole trapping time, and operating frequency, while negative ion spectra are relatively unaffected by changes in these parameters. The different response to instrumental tuning parameters is most likely attributable to the different spray and solvent conditions used for experiments in the positive and negative ion modes. The dependence of ions detected in the positive ion mode on tuning conditions is illustrated in Figure $1 \mathrm{a}, \mathrm{b}$, and $\mathrm{c}$. These ESI-MS spectra were acquired under three different ion accumulation conditions, but identical electrospray conditions. Measured and calculated masses of the most abundant ions observed in Figure 1a, b, and $c$ are given in Table 1. Although the measurements discussed in this paper cannot provide direct evidence for the structures of these ions since only chemical composition can be determined by accurate mass measurement, structural features such as those shown in Table 1 and throughout this report are suggested to aid the reader's understanding. It should be noted in this regard that certain structures could involve different atom oxidation states. Since based on $\mathrm{m} / \mathrm{z}$ values alone, charge state assignment for individual moieties cannot be made unambiguously, the convention used throughout this work is not to show charges on individual moieties in the complexes, as suggested and applied by Van Stipdonk et al. [21]. Thus, in this format, ions in which different uranyl oxidation states could be present are treated as equivalent.

Use of the higher quadrupole operating frequency $(1820 \mathrm{kHz})$, a relatively short quadrupole trapping pulse length of $2.881 \mathrm{~ms}$, and a hexapole trapping time of $1000 \mathrm{~ms}$ results in the detection of $\left[\left(\mathrm{UO}_{2}\right)\left(\mathrm{CH}_{3} \mathrm{O}\right)\right]^{+}$at $\mathrm{m} / \mathrm{z} 301$ (Figure 1a). The use of a lower operating frequency $(965 \mathrm{kHz})$ with a longer quadrupole trapping pulse length $(3.167 \mathrm{~ms})$ results in the detection of more complex adducts (Figure 1b). Here, a hexapole trapping time of $400 \mathrm{~ms}$ was used together with a two-step filament trapping in which the plate potential was first decreased from 10 to $4 \mathrm{~V}$ and then to $0 \mathrm{~V}$. The detected species include $\left[\left(\mathrm{UO}_{2}\right)\left(\mathrm{CH}_{3} \mathrm{O}\right)\left(\mathrm{CH}_{3} \mathrm{OH}\right)_{2}\right]^{+}$and $\left[\left(\mathrm{UO}_{2}\right)\left(\mathrm{NO}_{3}\right)\left(\mathrm{CH}_{3} \mathrm{OH}\right)_{2}\right]^{+}$at $m / z 365$ and $m / z 396$, respectively (Table 1). These ions have been previously characterized by Van Stipdonk et al. [18] in an ion trap mass spectrometer. The inset of Figure $1 \mathrm{~b}$ shows doublycharged species corresponding to the radical-containing ions of $\left[\left(\mathrm{UO}_{2}\right)\left(\mathrm{CH}_{3} \mathrm{O}\right)\left(\mathrm{CH}_{3} \mathrm{OH}\right)_{2}\right]^{2+\cdot}(\mathrm{m} / \mathrm{z}$ 182.5579, Table $1)$ and $\left[\left(\mathrm{UO}_{2}\right)\left(\mathrm{NO}_{3}\right)\left(\mathrm{CH}_{3} \mathrm{OH}\right)_{2}\right]^{2+\cdot}(\mathrm{m} / z$ 198). The highest mass ion in Figure $1 \mathrm{~b}$ appears at $\mathrm{m} / \mathrm{z} 790$ and can be assigned as $\left[\left(\mathrm{UO}_{2}\right)_{2}\left(\mathrm{NO}_{3}\right)_{3}\left(\mathrm{CH}_{3} \mathrm{OH}\right)_{2}\right]^{+}$. A related species, $\left[\left(\mathrm{UO}_{2}\right)_{2}\left(\mathrm{NO}_{3}\right)_{3}\left(\mathrm{H}_{2} \mathrm{O}\right)_{2}\right]^{+}$, has been detected by Van Stipdonk et al. [18]. Still more complex species were detected with the use of the lower frequency coil (965 $\mathrm{kHz}$ ), a significantly longer quadrupole trapping pulse length of $3.800 \mathrm{~ms}$, and a hexapole trapping time of 1000 ms (Figure 1c). In this case, only ions of $\mathrm{m} / \mathrm{z}>500$ appear in the spectrum. Ions with higher mass $(>700$ $\mathrm{Da})$ correspond to oligomeric uranyl nitrate species that can be characterized as having a general formula of $\left[\left(\mathrm{UO}_{2}\right)_{\mathrm{n}}(\mathrm{A})_{\mathrm{m}}\left(\mathrm{CH}_{3} \mathrm{OH}\right)_{\mathrm{s}}\right]^{+}$or $\left[\left(\mathrm{UO}_{2}\right)_{\mathrm{n}}(\mathrm{O})(\mathrm{A})_{\mathrm{m}}\left(\mathrm{CH}_{3} \mathrm{OH}\right)_{\mathrm{s}}\right]^{+}$ with $n=2-4, \mathrm{~m}=1-7$, and $\mathrm{s}=0$ or 1 . In both cases, A can be $\mathrm{OH}, \mathrm{NO}_{3}, \mathrm{CH}_{3} \mathrm{O}$ or a combination of these, although the formation of nitrate-containing species is clearly preferred (Table 1 for details).

The use of different tuning parameters is obviously important in gaining a more detailed understanding of the formation of gas-phase uranyl species during electrospray ionization. It is difficult to say whether the spectral sensitivity to tuning parameters is (1) due exclusively to possible ion-molecule reactions during the hexapole trapping time, or (2) related to the different hexapole/quadrupole transmittance of ions originally formed in the ESI process. (Note that external ESI source parameters were not changed during these measurements, i.e., the same spray was used, and that the spectra obtained by different tuning parameters were remarkably reproducible.) In any case, the variety of ions detected clearly indicates that the doubly charged $\mathrm{UO}_{2}^{2+}$ participates in rich reaction chemistry during the ESI process, forming numerous chemically- and structurally-distinct species. Given the lack of available information on the solvation of $\mathrm{UO}_{2}^{2+}$ in dilute solutions containing high concentrations of alcohols, it is difficult to say whether any of the detected ions are actual solution-phase species or if they are simply formed during the electrospray process. 
Table 1. Assignments for positive ions resulting from a solution $330 \mu \mathrm{M}$ in $\mathrm{UO}_{2}^{2+}$ and $660 \mu \mathrm{M}$ in $\mathrm{NO}_{3}^{-}$in a $66 \% \mathrm{CH}_{3} \mathrm{OH} / 33 \% \mathrm{H}_{2} \mathrm{O}$ (vol/vol) solvent system

\begin{tabular}{|c|c|c|c|}
\hline Nominal $m / z^{a}$ & Observed $\mathrm{m} / \mathrm{z}$ & Calculated $\mathrm{m} / \mathrm{z}$ & Assignment \\
\hline 301 & 301.0590 & 301.0590 & {$\left[\left(\mathrm{UO}_{2}\right)\left(\mathrm{CH}_{3} \mathrm{O}\right)\right]^{+}$} \\
\hline 183 & 182.5579 & 182.5557 & {$\left[\left(\mathrm{UO}_{2}\right)\left(\mathrm{CH}_{3} \mathrm{O}\right)\left(\mathrm{CH}_{3} \mathrm{OH}\right)_{2}\right]^{2+\cdot}$} \\
\hline 198 & 198.0424 & 198.0404 & {$\left[\left(\mathrm{UO}_{2}\right)\left(\mathrm{NO}_{3}\right)\left(\mathrm{CH}_{3} \mathrm{OH}\right)_{2}\right]^{2+\cdot}$} \\
\hline $365^{*}$ & 365.1114 & 365.1114 & {$\left[\left(\mathrm{UO}_{2}\right)\left(\mathrm{CH}_{3} \mathrm{O}\right)\left(\mathrm{CH}_{3} \mathrm{OH}\right)_{2}\right]^{+}$} \\
\hline 396 & 396.0797 & 396.0809 & {$\left[\left(\mathrm{UO}_{2}\right)\left(\mathrm{NO}_{3}\right)\left(\mathrm{CH}_{3} \mathrm{OH}\right)_{2}\right]^{+}$} \\
\hline 790 & 790.1009 & 790.0971 & {$\left[\left(\mathrm{UO}_{2}\right)_{2}\left(\mathrm{NO}_{3}\right)_{3}\left(\mathrm{CH}_{3} \mathrm{OH}\right)_{2}\right]^{+}$} \\
\hline $572 *$ & 572.0711 & 572.0711 & {$\left[\left(\cup_{2}\right)_{2}(\mathrm{O})_{2}\right]^{+}$} \\
\hline 587 & 587.0949 & 587.0945 & {$\left[\left(\mathrm{UO}_{2}\right)_{2}(\mathrm{O})\left(\mathrm{CH}_{3} \mathrm{O}\right)\right]^{+}$} \\
\hline 615 & & & unassigned \\
\hline 618 & 618.0639 & 618.0640 & {$\left[\left(\mathrm{UO}_{2}\right)_{2}(\mathrm{O})\left(\mathrm{NO}_{3}\right)\right]^{+}$} \\
\hline 726 & 726.0465 & 726.0447 & {$\left[\left(\mathrm{UO}_{2}\right)_{2}\left(\mathrm{NO}_{3}\right)_{3}\right]^{+}$} \\
\hline 981 & 981.1155 & 981.1108 & {$\left[\left(\mathrm{UO}_{2}\right)_{3}(\mathrm{O})\left(\mathrm{NO}_{3}\right)_{2}\left(\mathrm{CH}_{3} \mathrm{O}\right)\right]^{+}$} \\
\hline 1012 & 1012.0760 & 1012.0802 & {$\left[\left(\mathrm{UO}_{2}\right)_{3}(\mathrm{O})\left(\mathrm{NO}_{3}\right)_{3}\right]^{+}$} \\
\hline 1044 & 1044.1065 & 1044.1064 & {$\left[\left(\mathrm{UO}_{2}\right)_{3}(\mathrm{O})\left(\mathrm{NO}_{3}\right)_{3}\left(\mathrm{CH}_{3} \mathrm{OH}\right)\right]^{+}$} \\
\hline 1152 & 1152.0856 & 1152.0871 & {$\left[\left(\mathrm{UO}_{2}\right)_{3}\left(\mathrm{NO}_{3}\right)_{5}\left(\mathrm{CH}_{3} \mathrm{OH}\right)\right]^{+}$} \\
\hline 1406 & 1406.0990 & 1406.0964 & {$\left[\left(\mathrm{UO}_{2}\right)_{4}(\mathrm{O})\left(\mathrm{NO}_{3}\right)_{5}\right]^{+}$} \\
\hline 1469 & 1469.0967 & 1469.0921 & {$\left[\left(\mathrm{UO}_{2}\right)_{4}\left(\mathrm{NO}_{3}\right)_{6}(\mathrm{OH})\right]^{+}$} \\
\hline 1483 & 1483.1106 & 1483.1077 & {$\left[\left(\mathrm{UO}_{2}\right)_{4}\left(\mathrm{NO}_{3}\right)_{6}\left(\mathrm{CH}_{3} \mathrm{O}\right)\right]^{+}$} \\
\hline 1546 & 1546.1078 & 1546.1034 & {$\left[\left(\mathrm{UO}_{2}\right)_{4}\left(\mathrm{NO}_{3}\right)_{7}\left(\mathrm{CH}_{3} \mathrm{OH}\right)\right]^{+}$} \\
\hline 1800 & 1800.0585 & & unassigned \\
\hline
\end{tabular}

${ }^{a} \mathrm{~m} / \mathrm{z}$ Species denoted with * indicate ions used for calibration; ions of $\mathrm{m} / \mathrm{z}>900$ were calibrated using Ultramark 1621.

Table 2. Assignments for positive ions resulting from reaction of $\mathrm{D}_{2} \mathrm{O}$ with high-mass positive ions at selected $m / z$

\begin{tabular}{|c|c|c|c|}
\hline Nominal $m / z^{\mathrm{ab}}$ & Observed $\mathrm{m} / \mathrm{z}$ & Calculated $\mathrm{m} / \mathrm{z}$ & Assignment \\
\hline $1483 *$ & 1483.1077 & 1483.1077 & {$\left[\left(\mathrm{UO}_{2}\right)_{4}\left(\mathrm{NO}_{3}\right)_{6}\left(\mathrm{CH}_{3} \mathrm{O}\right)\right]^{+}$} \\
\hline 1502 & 1502.1260 & 1502.1246 & {$\left[\left(\mathrm{UO}_{2}\right)_{4}\left(\mathrm{NO}_{3}\right)_{6}\left(\mathrm{CH}_{3} \mathrm{O}\right)(\mathrm{HOD})\right]^{+}$} \\
\hline 1503 & 1503.1322 & 1503.1309 & {$\left[\left(\mathrm{UO}_{2}\right)_{4}\left(\mathrm{NO}_{3}\right)_{6}\left(\mathrm{CH}_{3} \mathrm{O}\right)\left(\mathrm{D}_{2} \mathrm{O}\right)\right]^{+}$} \\
\hline 1519 & 1519.1600 & 1519.1592 & {$\left[\left(\mathrm{UO}_{2}\right)_{4}\left(\mathrm{NO}_{3}\right)_{6}\left(\mathrm{CH}_{3} \mathrm{O}\right)\left(\mathrm{CD}_{3} \mathrm{OD}\right)\right]^{+}$} \\
\hline 1012 & Not obs. & 1012.0802 & {$\left[\left(\mathrm{UO}_{2}\right)_{3}(\mathrm{O})\left(\mathrm{NO}_{3}\right)_{3}\right]^{+}$} \\
\hline 1051 & 1051.1227 & 1051.1202 & {$\left[\left(\mathrm{UO}_{2}\right)_{3}(\mathrm{O})\left(\mathrm{NO}_{3}\right)_{3}\left(\mathrm{D}_{2} \mathrm{O}\right)(\mathrm{HOD})\right]^{+}$} \\
\hline 1052 & 1052.1268 & 1052.1264 & {$\left[\left(\mathrm{UO}_{2}\right)_{3}(\mathrm{O})\left(\mathrm{NO}_{3}\right)_{3}\left(\mathrm{D}_{2} \mathrm{O}\right)_{2}\right]^{+}$} \\
\hline 1068 & 1068.1597 & 1068.1546 & {$\left[\left(\mathrm{UO}_{2}\right)_{3}(\mathrm{O})\left(\mathrm{NO}_{3}\right)_{3}\left(\mathrm{D}_{2} \mathrm{O}\right)\left(\mathrm{CD}_{3} \mathrm{OD}\right)\right]^{+}$} \\
\hline 1069 & 1069.1322 & 1069.1307 & {$\left[\left(\mathrm{UO}_{2}\right)_{3}(\mathrm{O})\left(\mathrm{NO}_{3}\right)_{3}(\mathrm{HOD})_{3}\right]^{+}$} \\
\hline 1070 & 1070.1365 & 1070.1370 & {$\left[\left(\mathrm{UO}_{2}\right)_{3}(\mathrm{O})\left(\mathrm{NO}_{3}\right)_{3}\left(\mathrm{D}_{2} \mathrm{O}\right)(\mathrm{HOD})_{2}\right]^{+}$} \\
\hline 1071 & 1071.1410 & 1071.1433 & {$\left[\left(\mathrm{UO}_{2}\right)_{3}(\mathrm{O})\left(\mathrm{NO}_{3}\right)_{3}\left(\mathrm{D}_{2} \mathrm{O}\right)_{2}(\mathrm{HOD})\right]^{+}$} \\
\hline $1072 *$ & 1072.1496 & 1072.1496 & {$\left[\left(\mathrm{UO}_{2}\right)_{3}(\mathrm{O})\left(\mathrm{NO}_{3}\right)_{3}\left(\mathrm{D}_{2} \mathrm{O}\right)_{3}\right]^{+}$} \\
\hline 1086 & 1086.1672 & 1086.1652 & {$\left[\left(\mathrm{UO}_{2}\right)_{3}(\mathrm{O})\left(\mathrm{NO}_{3}\right)_{3}\left(\mathrm{D}_{2} \mathrm{O}\right)(\mathrm{HOD})\left(\mathrm{CD}_{3} \mathrm{OH}\right)\right]^{+}$} \\
\hline 1087 & 1087.1720 & 1087.1715 & {$\left[\left(\mathrm{UO}_{2}\right)_{3}(\mathrm{O})\left(\mathrm{NO}_{3}\right)_{3}\left(\mathrm{D}_{2} \mathrm{O}\right)_{2}\left(\mathrm{CD}_{3} \mathrm{OH}\right)\right]^{+}$} \\
\hline 1088 & 1088.1800 & 1088.1778 & {$\left[\left(\mathrm{UO}_{2}\right)_{3}(\mathrm{O})\left(\mathrm{NO}_{3}\right)_{3}\left(\mathrm{D}_{2} \mathrm{O}\right)_{2}\left(\mathrm{CD}_{3} \mathrm{OD}\right)\right]^{+}$} \\
\hline 1103 & 1103.2029 & 1103.1997 & {$\left[\left(\mathrm{UO}_{2}\right)_{3}(\mathrm{O})\left(\mathrm{NO}_{3}\right)_{3}\left(\mathrm{D}_{2} \mathrm{O}\right)\left(\mathrm{CD}_{3} \mathrm{OD}\right)\left(\mathrm{CD}_{3} \mathrm{OH}\right)\right]^{+}$} \\
\hline 1104 & 1104.2091 & 1104.2060 & {$\left[\left(\mathrm{UO}_{2}\right)_{3}(\mathrm{O})\left(\mathrm{NO}_{3}\right)_{3}\left(\mathrm{D}_{2} \mathrm{O}\right)\left(\mathrm{CD}_{3} \mathrm{OD}\right)_{2}\right]^{+}$} \\
\hline $618^{*}$ & 618.0640 & 618.0640 & {$\left[\left(\mathrm{UO}_{2}\right)_{2}(\mathrm{O})\left(\mathrm{NO}_{3}\right)\right]^{+}$} \\
\hline 637 & 637.0816 & 637.0808 & {$\left[\left(\mathrm{UO}_{2}\right)_{2}(\mathrm{O})\left(\mathrm{NO}_{3}\right)(\mathrm{HOD})\right]^{+}$} \\
\hline 638 & 638.0874 & 638.0871 & {$\left[\left(\mathrm{UO}_{2}\right)_{2}(\mathrm{O})\left(\mathrm{NO}_{3}\right)\left(\mathrm{D}_{2} \mathrm{O}\right)\right]^{+}$} \\
\hline 654 & 654.1172 & 654.1153 & {$\left[\left(\mathrm{UO}_{2}\right)_{2}(\mathrm{O})\left(\mathrm{NO}_{3}\right)\left(\mathrm{CD}_{3} \mathrm{OD}\right)\right]^{+}$} \\
\hline 656 & 656.0974 & 656.0976 & {$\left[\left(\mathrm{UO}_{2}\right)_{2}(\mathrm{O})\left(\mathrm{NO}_{3}\right)(\mathrm{HOD})_{2}\right]^{+}$} \\
\hline 657 & 657.1031 & 657.1039 & {$\left[\left(\mathrm{UO}_{2}\right)_{2}(\mathrm{O})\left(\mathrm{NO}_{3}\right)\left(\mathrm{D}_{2} \mathrm{O}\right)(\mathrm{HOD})\right]^{+}$} \\
\hline 658 & 658.1086 & 658.1102 & {$\left[\left(\mathrm{UO}_{2}\right)_{2}(\mathrm{O})\left(\mathrm{NO}_{3}\right)\left(\mathrm{D}_{2} \mathrm{O}\right)_{2}\right]^{+}$} \\
\hline 676 & 676.1218 & 676.1208 & {$\left[\left(\mathrm{UO}_{2}\right)_{2}(\mathrm{O})\left(\mathrm{NO}_{3}\right)\left(\mathrm{D}_{2} \mathrm{O}\right)(\mathrm{HOD})_{2}\right]^{+}$} \\
\hline 677 & 677.1289 & 677.1270 & {$\left[\left(\mathrm{UO}_{2}\right)_{2}(\mathrm{O})\left(\mathrm{NO}_{3}\right)\left(\mathrm{D}_{2} \mathrm{O}\right)_{2}(\mathrm{HOD})\right]^{+}$} \\
\hline 678 & 678.1347 & 678.1333 & {$\left[\left(\mathrm{UO}_{2}\right)_{2}(\mathrm{O})\left(\mathrm{NO}_{3}\right)\left(\mathrm{D}_{2} \mathrm{O}\right)_{3}\right]^{+}$} \\
\hline 693 & 693.1543 & 693.1552 & {$\left[\left(\mathrm{UO}_{2}\right)_{2}(\mathrm{O})\left(\mathrm{NO}_{3}\right)\left(\mathrm{D}_{2} \mathrm{O}\right)_{2}\left(\mathrm{CD}_{3} \mathrm{OH}\right)\right]^{+}$} \\
\hline 694 & 694.1596 & 694.1615 & {$\left[\left(\mathrm{UO}_{2}\right)_{2}(\mathrm{O})\left(\mathrm{NO}_{3}\right)\left(\mathrm{D}_{2} \mathrm{O}\right)_{2}\left(\mathrm{CD}_{3} \mathrm{OD}\right)\right]^{+}$} \\
\hline 574 & 574.0835 & 574.0852 & {$\left[\left(\mathrm{UO}_{2}\right)_{2}(\mathrm{O})(\mathrm{OD})\right]^{+}$} \\
\hline $587^{*}$ & 587.0945 & 587.0945 & {$\left[\left(\mathrm{UO}_{2}\right)_{2}(\mathrm{O})\left(\mathrm{CH}_{3} \mathrm{O}\right)\right]^{+}$} \\
\hline 590 & 590.1139 & 590.1164 & {$\left[(\mathrm{UO})_{2}(\mathrm{OH})\left(\mathrm{CH}_{3} \mathrm{OD}\right)\right]^{+}$} \\
\hline 606 & 606.1105 & 606.1114 & {$\left[\left(\mathrm{UO}_{2}\right)_{2}(\mathrm{O})\left(\mathrm{CH}_{3} \mathrm{O}\right)(\mathrm{HOD})\right]^{+}$} \\
\hline 607 & 607.1165 & 607.1176 & {$\left[\left(\mathrm{UO}_{2}\right)_{2}(\mathrm{O})\left(\mathrm{CH}_{3} \mathrm{O}\right)\left(\mathrm{D}_{2} \mathrm{O}\right)\right]^{+}$} \\
\hline 610 & 610.1368 & 610.1396 & {$\left[\left(\mathrm{UO}_{2}\right)_{2}(\mathrm{OH})\left(\mathrm{CH}_{3} \mathrm{OD}\right)\left(\mathrm{D}_{2} \mathrm{O}\right)\right]^{+}$} \\
\hline 626 & 626.1324 & 626.1345 & {$\left[\left(\mathrm{UO}_{2}\right)_{2}(\mathrm{O})\left(\mathrm{CH}_{3} \mathrm{O}\right)\left(\mathrm{D}_{2} \mathrm{O}\right)(\mathrm{HOD})\right]^{+}$} \\
\hline 627 & 627.1383 & 627.1408 & {$\left[\left(\mathrm{UO}_{2}\right)_{2}(\mathrm{O})\left(\mathrm{CH}_{3} \mathrm{O}\right)\left(\mathrm{D}_{2} \mathrm{O}\right)_{2}\right]^{+}$} \\
\hline
\end{tabular}

${ }^{\text {a }}$ Reacted ions in bold.

${ }^{\mathrm{b}} \mathrm{m} / \mathrm{z}$ Species denoted with * indicate ions used for calibration. 

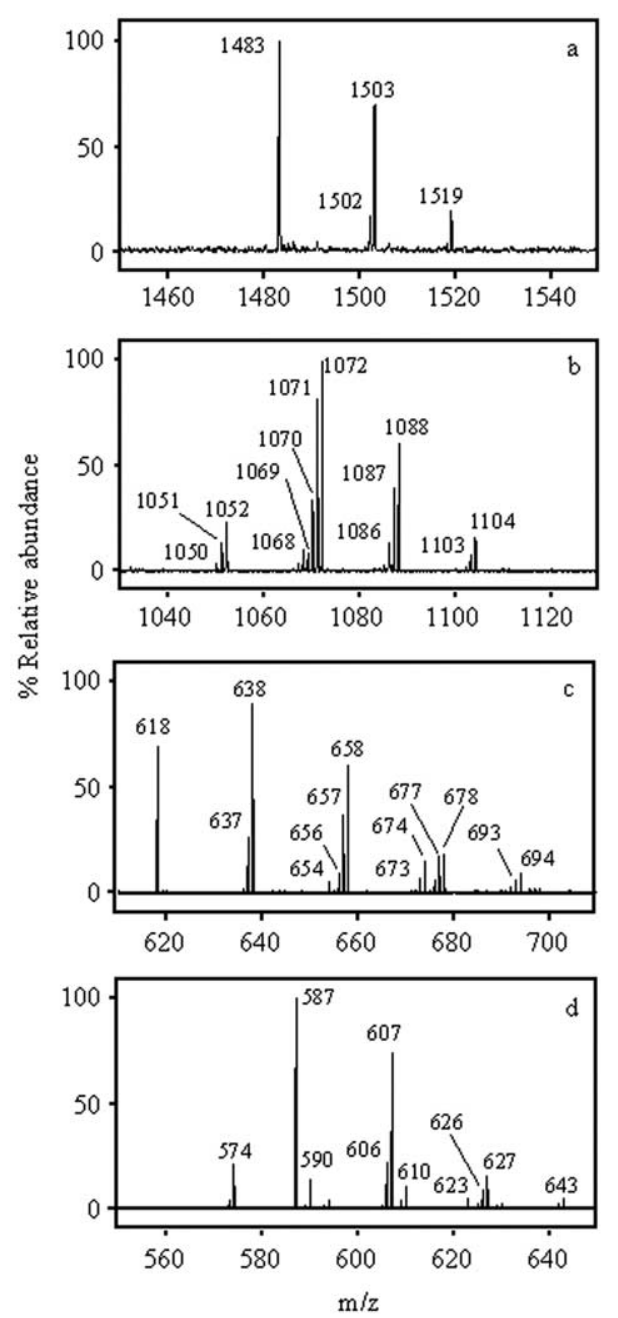

Figure 2. Ions produced by ion-molecule reactions between $\mathrm{D}_{2} \mathrm{O}$ and high-mass positive ions at (a) $\mathrm{m} / \mathrm{z} 1483$, (b) $\mathrm{m} / \mathrm{z} 1012$, (c) $\mathrm{m} / \mathrm{z}$ 618 , and (d) $\mathrm{m} / \mathrm{z}$ 587. Reaction time was $10 \mathrm{~s}$ in each case with a $\mathrm{D}_{2} \mathrm{O}$ pressure of $2.4 \times 10^{-8}$ torr.

The MS/MS fragmentation patterns of the low mass species $(<500 \mathrm{Da})$ in Figure 1a and $\mathrm{b}$ have been previously discussed [18]. The fragmentation patterns of the higher mass species shown in Figure 1c are discussed below.

\section{Ion-Molecule Reactions in the Gas-Phase with $\mathrm{D}_{2} \mathrm{O}$ and $\mathrm{CD}_{3} \mathrm{OD}$ as Reaction Partners}

Ion-molecule reactions in the gas-phase have been used extensively to gain information on the chemistry and reactivity of metals in coordination complexes, as well as the structures of these metal-ligand complexes [25-34]. In our studies, $\mathrm{D}_{2} \mathrm{O}$ and adventitious $\mathrm{CD}_{3} \mathrm{OD}$ were used as neutral reaction partners with several of the more abundant ions in Figure $1 \mathrm{c}$ to assess the degree of coordinative unsaturation of the uranyl in each of the selected species. Representative spectra of the reaction region obtained after reaction of the major ions in Figure $1 \mathrm{c}$ with $\mathrm{D}_{2} \mathrm{O}$ for $10 \mathrm{~s}$ at 2.4 $\times 10^{-8}$ torr are shown in Figure $2 \mathrm{a}, \mathrm{b}, \mathrm{c}$, and $\mathrm{d}$. Introduction of $\mathrm{D}_{2} \mathrm{O}$ into the FT-ICR cell results not only in reaction products but also in some fragmentation of the selected ion. As an example, a representative full-scale spectrum of products appearing upon reaction of $\left[\left(\mathrm{UO}_{2}\right)_{3}(\mathrm{O})\left(\mathrm{NO}_{3}\right)_{3}\right]^{+}\left(\mathrm{m} / z\right.$ 1012) with $\mathrm{D}_{2} \mathrm{O}$ is shown in Figure 3. Assignments and exact mass values for the ions shown in Figure 2a, b, c, and d and Figure 3 are provided in Table 2.

Upon examination of the spectra in Figure 2a, b, c, and $\mathrm{d}$ and the data in Table 2, two patterns emerge. First, the selected ion may undergo a simple addition of one or more $\mathrm{D}_{2} \mathrm{O}$ ligands, and the number of $\mathrm{D}_{2} \mathrm{O}$ ligands accepted appears to depend on the structure of the ion. $\mathrm{H}_{2} \mathrm{O}$ and $\mathrm{HOD}$ additions also occur, indicating the presence of residual $\mathrm{H}_{2} \mathrm{O}$ in the FT-ICR cell that participates in $\mathrm{H} / \mathrm{D}$ exchange reactions with $\mathrm{D}_{2} \mathrm{O}$. Additions of $\mathrm{CD}_{3} \mathrm{OD}$ are also apparent and must result from residual $\mathrm{CD}_{3} \mathrm{OD}$ in the analyzer region of the system or the exchange reagent reservoir/pulsed leak system.

The highest mass ion selected, $\left[\left(\mathrm{UO}_{2}\right)_{4}\left(\mathrm{NO}_{3}\right)_{6}\right.$ $\left.\left(\mathrm{CH}_{3} \mathrm{O}\right)\right]^{+}$at $\mathrm{m} / \mathrm{z} 1483$, undergoes only a simple single addition of $\mathrm{HOD}, \mathrm{D}_{2} \mathrm{O}$, or $\mathrm{CD}_{3} \mathrm{OD}$, forming ions at $\mathrm{m} / \mathrm{z}$ 1502, 1503, and 1519, respectively (Figure 2a). These products are also the only ones formed after a $60 \mathrm{~s} \mathrm{D}_{2} \mathrm{O}$ reaction time (data not shown). Although based on these data alone the structure of this ion cannot be definitely determined, its lack of reactivity is one indication that the uranyl centers are as fully coordinatively saturated as possible except for one site; therefore, the ion is unable to accept more than one ligand at this pressure. Although the full spectrum is not shown here, an ion at $\mathrm{m} / \mathrm{z} 270$ also appears in the spectrum. This ion corresponds to $\mathrm{UO}_{2}^{+}$, and it is observed in the fragmentation spectra of other positively charged uranyl-containing species as well. The intensity of this ion is an indicator of the extent of ion fragmentation that must be related to the stability of a given uranyl complex. Upon reaction of $\left[\left(\mathrm{UO}_{2}\right)_{4}\left(\mathrm{NO}_{3}\right)_{6}\left(\mathrm{CH}_{3} \mathrm{O}\right)\right]^{+}(\mathrm{m} / \mathrm{z}$ 1483) with $\mathrm{D}_{2} \mathrm{O}$, the $\mathrm{UO}_{2}^{+}$peak is small, indicating that the ion is relatively stable and does not fragment extensively. Presumably, the four $\mathrm{UO}_{2}^{2+}$ groups are coordinatively saturated by nitrate in an oligomeric structure, although it is difficult to predict the structure of this ion based only on its presence and stability.

Another "nonreactive" ion is $\left[\left(\mathrm{UO}_{2}\right)_{3}\left(\mathrm{NO}_{3}\right)_{5}\right.$ $\left.\left(\mathrm{CH}_{3} \mathrm{OH}\right)\right]^{+}$at $m / z$ 1152. This ion apparently fragments on collision with $\mathrm{D}_{2} \mathrm{O}$, but does not add a $\mathrm{D}_{2} \mathrm{O}$ ligand, indicating again that all coordination sites are filled (spectrum not shown).

$\left[\left(\mathrm{UO}_{2}\right)_{3}(\mathrm{O})\left(\mathrm{NO}_{3}\right)_{3}\right]^{+}$at $\mathrm{m} / \mathrm{z} 1012$ is significantly more reactive than $\left[\left(\mathrm{UO}_{2}\right)_{4}\left(\mathrm{NO}_{3}\right)_{6}\left(\mathrm{CH}_{3} \mathrm{O}\right)\right]^{+}$and undergoes addition of up to three more ligands (Figure 2b, Table 2). After a $\mathrm{D}_{2} \mathrm{O}$ reaction time of $10 \mathrm{~s}$, the parent ion has completely disappeared. The greater reactivity of this ion at this pressure is an indication that the uranyl groups in $\left[\left(\mathrm{UO}_{2}\right)_{3}(\mathrm{O})\left(\mathrm{NO}_{3}\right)_{3}\right]^{+}$are coordinatively less saturated than those in $\left[\left(\mathrm{UO}_{2}\right)_{4}\left(\mathrm{NO}_{3}\right)_{6}\left(\mathrm{CH}_{3} \mathrm{O}\right)\right]^{+}(\mathrm{m} / \mathrm{z}$ 


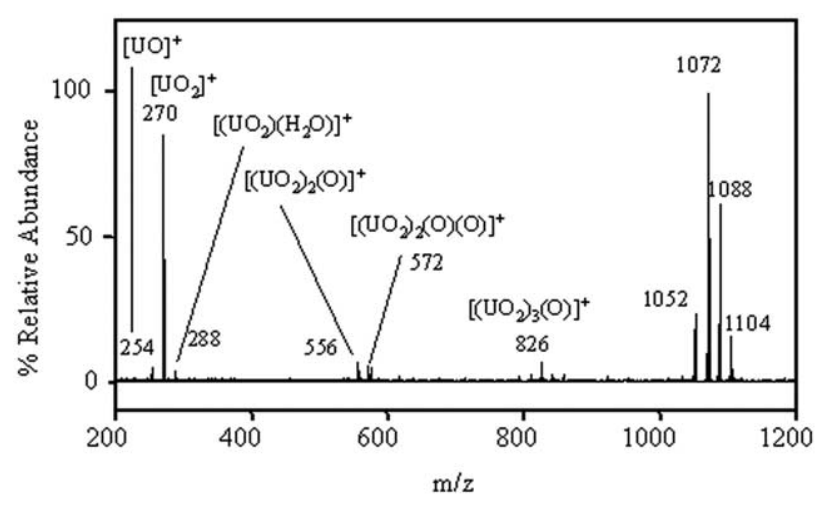

Figure 3. Representative mass spectrum showing fragmentation of $m / z 1012$ and its reaction products after $10 \mathrm{~s}$ reaction with $\mathrm{D}_{2} \mathrm{O}$ $\left(2.4 \times 10^{-8}\right.$ torr $)$.

1483), and implies structural differences between the two ions. $\left[\left(\mathrm{UO}_{2}\right)_{3}(\mathrm{O})\left(\mathrm{NO}_{3}\right)_{3}\right]^{+}$contains what may be a bridging oxygen ligand between the three uranyl groups. The addition of three $\mathrm{D}_{2} \mathrm{O}$ or $\mathrm{CD}_{3} \mathrm{OD}$ ligands suggests that each uranyl moiety can accommodate only one more ligand in addition to the nitrates, which probably have bidentate coordination to the uranyl. $\left[\left(\mathrm{UO}_{2}\right)_{3}(\mathrm{O})\left(\mathrm{NO}_{3}\right)_{3}\right]^{+}$also undergoes more extensive fragmentation upon reaction with $\mathrm{D}_{2} \mathrm{O}$ (Figure 3) than does $\left[\left(\mathrm{UO}_{2}\right)_{4}\left(\mathrm{NO}_{3}\right)_{6}\left(\mathrm{CH}_{3} \mathrm{O}\right)\right]^{+}$, which is evidence that it is the more fragile species.

It is interesting to compare the reactions of $\left[\left(\mathrm{UO}_{2}\right)_{2}(\mathrm{O})\left(\mathrm{CH}_{3} \mathrm{O}\right)\right]^{+}$at $\mathrm{m} / z 587$ and $\left[\left(\mathrm{UO}_{2}\right)_{2}(\mathrm{O})\left(\mathrm{NO}_{3}\right)\right]^{+}$ at $m / z 618$ with $\mathrm{D}_{2} \mathrm{O}$, as these ions differ only in whether they contain a methoxy or a nitrate ligand (Figure 2c and $\mathrm{d}$, Table 2). Interestingly, $\left[\left(\mathrm{UO}_{2}\right)_{2}(\mathrm{O})\left(\mathrm{NO}_{3}\right)\right]^{+}$adds three ligands after a $10 \mathrm{~s}$ reaction time, while $\left[\left(\mathrm{UO}_{2}\right)_{2}(\mathrm{O})\left(\mathrm{CH}_{3} \mathrm{O}\right)\right]^{+}$only accommodates two. DFT calculations indicate that nitrate can coordinate to uranyl in either a bidentate or monodentate configuration in gas-phase $\left[\left(\mathrm{UO}_{2}\right)\left(\mathrm{NO}_{3}\right)\left(\mathrm{H}_{2} \mathrm{O}\right)_{3}\right]^{+}$complexes [19]. A monodentate bridging nitrate ligand in $\left[\left(\mathrm{UO}_{2}\right)_{2}(\mathrm{O})\left(\mathrm{NO}_{3}\right)\right]^{+}$ could, in theory, provide a free nonbridging oxygen atom of the nitrate with which additional $\mathrm{D}_{2} \mathrm{O}$ or $\mathrm{CD}_{3} \mathrm{OD}$ could interact through hydrogen bonding. Under this scenario, a free oxygen atom is not available for such hydrogen bonding when methoxy replaces nitrate in the complex, and therefore, fewer ligands can be added. Interestingly, $\left[\left(\mathrm{UO}_{2}\right)_{2}(\mathrm{O})\left(\mathrm{NO}_{3}\right)\right]^{+}$fragments less extensively than $\left[\left(\mathrm{UO}_{2}\right)_{2}(\mathrm{O})\left(\mathrm{CH}_{3} \mathrm{O}\right)\right]^{+}$upon reaction with $\mathrm{D}_{2} \mathrm{O}$, indicating that it is the more stable ion (spectrum not shown). One other major difference between the two ions is that $\left[\left(\mathrm{UO}_{2}\right)_{2}(\mathrm{O})\left(\mathrm{CH}_{3} \mathrm{O}\right)\right]^{+}$undergoes a methoxy/deuteroxy replacement reaction, forming $\left[\left(\mathrm{UO}_{2}\right)_{2}(\mathrm{O})(\mathrm{OD})\right]^{+}$at $\mathrm{m} / \mathrm{z} 574$ (Figure $2 \mathrm{~d}$ ). A similar replacement is not seen for $\left[\left(\mathrm{UO}_{2}\right)_{2}(\mathrm{O})\left(\mathrm{NO}_{3}\right)\right]^{+}$consistent with the above contention of its greater stability.

As mentioned above, fragmentation of the selected ions occurs during reaction with $\mathrm{D}_{2} \mathrm{O}$, indicating that these species are fragile. The full-scale spectrum of $\left[\left(\mathrm{UO}_{2}\right)_{3}(\mathrm{O})\left(\mathrm{NO}_{3}\right)_{3}\right]^{+}\left(\mathrm{m} / \mathrm{z}\right.$ 1012) after reaction with $\mathrm{D}_{2} \mathrm{O}$ is shown in Figure 3 as an example. Characteristic ions appearing in all reaction spectra include $\mathrm{UO}^{+}$at $\mathrm{m} / \mathrm{z}$ 254, $\mathrm{UO}_{2}^{+}$at $\mathrm{m} / \mathrm{z} 270$, and $\left[\left(\mathrm{UO}_{2}\right) \mathrm{H}_{2} \mathrm{O}\right]^{+}$at $\mathrm{m} / z 288$ or $\left[\left(\mathrm{UO}_{2}\right) \mathrm{OH}\right]^{+}$at $m / z$ 287. $\left[\left(\mathrm{UO}_{2}\right)_{2}(\mathrm{O})\right]^{+}$and $\left[\left(\mathrm{UO}_{2}\right)_{2}(\mathrm{O})_{2}\right]^{+}$, which appear at $m / z 556$ and 572 , respectively, are also seen in the MS/MS spectra obtained using sustained off-resonance ionization collision-induced dissociation (SORI-CID) with $\mathrm{N}_{2}$. The ion $\left[\left(\mathrm{UO}_{2}\right)_{3}(\mathrm{O})\right]^{+}$at $\mathrm{m} / z 826$ is unique to the $\mathrm{D}_{2} \mathrm{O}$ reaction spectrum of $\left[\left(\mathrm{UO}_{2}\right)_{3}(\mathrm{O})\left(\mathrm{NO}_{3}\right)_{3}\right]^{+}$, and may be evidence for oxygen bridging between three reduced uranyl groups. However, $\left[\left(\mathrm{UO}_{2}\right)_{2}(\mathrm{O})\right]^{+}, \quad\left[\left(\mathrm{UO}_{2}\right)_{2}(\mathrm{O})_{2}\right]^{+}$and $\left[\left(\mathrm{UO}_{2}\right)_{3}(\mathrm{O})\right]^{+}$ have also been reported from fast atom bombardment studies of inorganic and organic uranyl complexes [35-38], and could result from rearrangements occurring upon fragmentation.

Other ions in Figure 3 are due to formal addition of D or exchange of $\mathrm{D}^{+}$for $\mathrm{H}^{+}$in $\mathrm{OH}^{-}$to yield $\mathrm{OD}^{-}$, giving the assignments of $\left[\left(\mathrm{UO}_{2}\right) \mathrm{D}\right]^{+\cdot}(\mathrm{m} / \mathrm{z} 272)$ (not visible in spectrum), $\left[\left(\mathrm{UO}_{2}\right)_{2}(\mathrm{OD})\right]^{+}(\mathrm{m} / \mathrm{z} 558)$, and $\left[\left(\mathrm{UO}_{2}\right)_{2}(\mathrm{O})(\mathrm{OD})\right]^{+}(\mathrm{m} / \mathrm{z} 574)$. The appearance of deuterium in these ions is interesting, since it has been proposed previously that hydrated $\mathrm{UO}_{2}^{2+}$ in solution abstracts a hydrogen atom from water during photolysis, ultimately forming $\mathrm{UO}_{2}^{+}$and $\mathrm{H}^{+}$along with an $\mathrm{OH}$ radical [39]. A similar solution reaction has been proposed with $\mathrm{CH}_{3} \mathrm{OH}$ [39].

Despite the observed differences in stability of these oligomeric uranyl ions, clearly they add ligands only up to the point of coordinative saturation of the uranyl centers at the applied $\mathrm{D}_{2} \mathrm{O}$ pressure. Interestingly, as shown by the data in Table 2, for ions containing two, three or four uranyl centers, coordinative saturation is achieved with fewer ligands per uranyl center than observed previously for ions with a single uranyl center [18]. This observation clearly supports the presence of bridging ligands in ions containing multiple uranyl centers that are not possible in ions containing a single uranyl center.

As demonstrated above, most of the selected ions react extensively with $\mathrm{D}_{2} \mathrm{O}$. It is desirable, therefore, to determine how readily the "naked" $\mathrm{UO}_{2}^{+}$singly charged cation $(m / z 270)$ reacts with $\mathrm{D}_{2} \mathrm{O}$ and $\mathrm{CD}_{3} \mathrm{OD}$ in the FT-ICR cell. In both experiments, the ion-molecule reaction times were the same as for $m / z 301$ (180 s) but the pressures were slightly different $\left(2.4 \times 10^{-8}\right.$ torr for $\mathrm{D}_{2} \mathrm{O}$ and $4.8 \times 10^{-8}$ torr for $\left.\mathrm{CD}_{3} \mathrm{OD}\right) . \mathrm{UO}_{2}^{+}$reacts readily with $\mathrm{CD}_{3} \mathrm{OD}$ by adding three and four $\mathrm{CD}_{3} \mathrm{OD}$ molecules leading to the $\left[\left(\mathrm{UO}_{2}\right)\left(\mathrm{CH}_{3} \mathrm{OD}\right)_{3}\right]^{+}$ion at $\mathrm{m} / \mathrm{z}$ 378 and the $\left.\left[\left(\mathrm{UO}_{2}\right)\left(\mathrm{CH}_{3} \mathrm{OD}\right)_{4}\right)\right]^{+}$ion at $\mathrm{m} / \mathrm{z} 414$ (spectrum not shown). In contrast, no $\mathrm{D}_{2} \mathrm{O}$ addition was observed when $\mathrm{D}_{2} \mathrm{O}$ was used as a reaction partner. The absence of adducts with $\mathrm{D}_{2} \mathrm{O}$ does not necessarily mean that addition reactions do not occur. These adducts could easily fragment under these relatively low-pressure conditions because of the lack of efficient collisional cooling. This explanation is further supported by the observation of $\left[\mathrm{UO}_{2}\left(\mathrm{H}_{2} \mathrm{O}\right)_{3}\right]^{+}$and $\left[\mathrm{UO}_{2}\left(\mathrm{H}_{2} \mathrm{O}\right)_{4}\right]^{+}$in ion trap studies at higher partial pressures of water [17].

Another interesting difference is that doubly- 

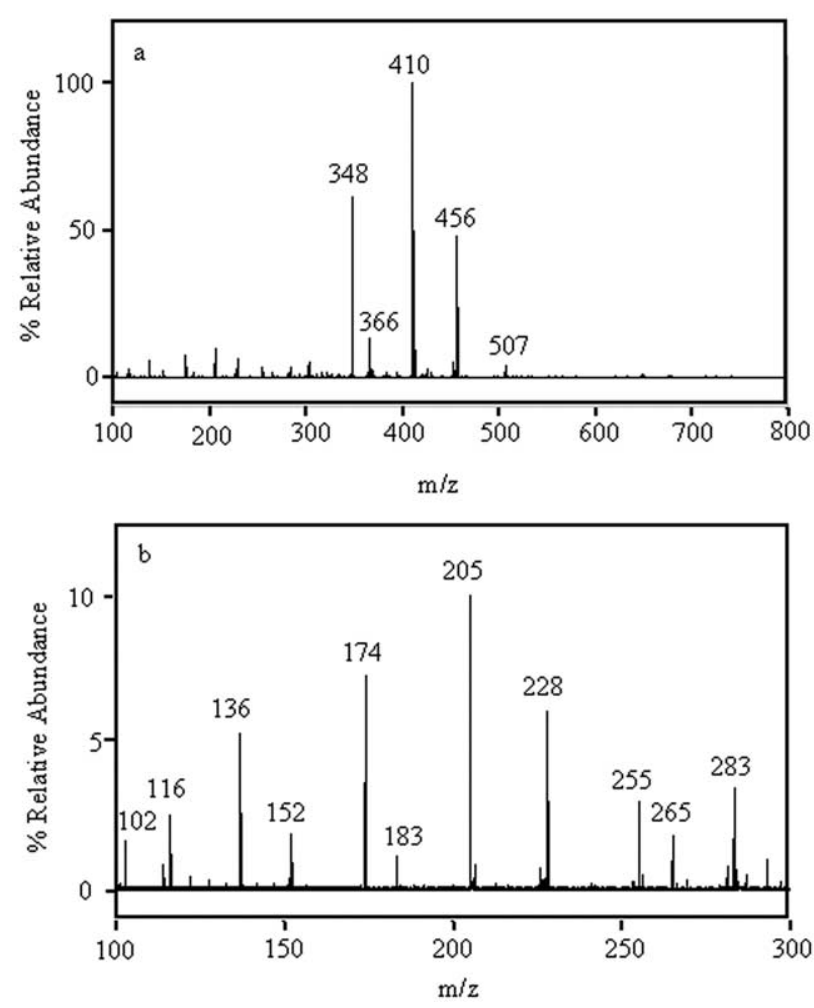

Figure 4. Negative ion ESI mass spectra of a solution containing $50 \mu \mathrm{M} \mathrm{UO}_{2}^{2+}$ and $100 \mu \mathrm{M} \mathrm{NO}_{3}^{-}$in $95 \% \mathrm{CH}_{3} \mathrm{OH} / 5 \% \mathrm{H}_{2} \mathrm{O}$ (vol/vol). (a) Full-scale with singly-charged ions indicated. (b) Expanded view of low mass region showing multiply-charged ions.

charged $\mathrm{UO}_{2}^{2+}(m / z$ 135) appears in the spectrum after reaction with $\mathrm{D}_{2} \mathrm{O}$, but is absent when $\mathrm{CD}_{3} \mathrm{OD}$ is used (spectra not shown). The doubly-charged $\mathrm{UO}_{2}^{2+}$ observed at $m / z 135$ in the presence of $\mathrm{D}_{2} \mathrm{O}$ can result from $\mathrm{DO}$ radical addition followed by $\mathrm{DO}^{-}$anion elimination as suggested earlier [39]. The absence of doubly-charged $\mathrm{UO}_{2}^{2+}$ in the spectrum resulting from reaction with $\mathrm{CD}_{3} \mathrm{OD}$ indicates that a similar process does not occur with the methoxy radical.

In addition to conventional methanol adducts, ions associated with the addition of $\mathrm{CD}_{3} \mathrm{OCD}_{3}$ are also observed. The most prominent of these are the $\left[\left(\mathrm{UO}_{2}\right)(\mathrm{D})\left(\mathrm{CD}_{3} \mathrm{OD}\right)\left(\mathrm{CD}_{3} \mathrm{OCD}_{3}\right)_{2}\right]^{+}$at $\mathrm{m} / \mathrm{z} 412$ and the $\left[\left(\mathrm{UO}_{2}\right)\left(\mathrm{CD}_{3} \mathrm{OD}\right)_{2}\left(\mathrm{CD}_{3} \mathrm{OCD}_{3}\right)_{2}\right]^{+}$at $m / z 446$. The perdeuterodimethyl ether can form either in the cell at the partial pressure of $\mathrm{CD}_{3} \mathrm{OD}$ used for these studies $(\sim 4.8$ $\times 10^{-8}$ torr) [40,41] or at the uranyl ("catalytic") centers. A more detailed investigation of the $\mathrm{m} / \mathrm{z} 411-$ 414 region also reveals another ion at $\mathrm{m} / \mathrm{z} 412$ corresponding to $\left[\left(\mathrm{UO}_{2}\right)\left(\mathrm{CD}_{3} \mathrm{O}\right)\left(\mathrm{CD}_{3} \mathrm{OD}\right)_{3}\right]^{+}$.

\section{Negative Ions Containing Uranyl and Nitrate Generated by ESI}

Alkali and transition-metal cations are known to form complexes with nitrate when an excess of $\mathrm{NO}_{3}^{-}$is present in solution $[42,43]$. Generally, these complexes are of the form $\left[\left(\mathrm{UO}_{2}\right)\left(\mathrm{NO}_{3}\right)_{\mathrm{m}}\right]^{-}(\mathrm{m}=1-3)$, although the formation of oxo-nitrate $\left(\left[\left(\mathrm{UO}_{2}\right)(\mathrm{O})\left(\mathrm{NO}_{3}\right)_{\mathrm{m}}\right]^{-}(\mathrm{m}=1-2)\right.$ and hydroxy-nitrate $\left(\left[\left(\mathrm{UO}_{2}\right)(\mathrm{OH})_{\mathrm{n}}\left(\mathrm{NO}_{3}\right)_{\mathrm{m}}\right]^{-}(n=1-2, \mathrm{~m}\right.$ $=0-1$ ) products are also observed [43]. In agreement with these previous observations, we have also detected uranyl nitrate and oxo-nitrate gas-phase complexes in the negative ion mode (Figure 4a). An expanded view of the low mass region is shown in Figure 4b. Measured and calculated masses of the ions observed are given in Table 3. These negative ion spectra were acquired using a quadrupole operating frequency of $1820 \mathrm{kHz}$ with a quadrupole trapping pulse length of $3.200 \mathrm{~ms}$. In contrast to the behavior observed with positive ions, changing these ion accumulation parameters did not affect the negative ion species detected.

The three most intense ions in Figure 4a appear at $\mathrm{m} / \mathrm{z} 348,410$ and 456 . The ion at $\mathrm{m} / \mathrm{z} 456$ is assigned to $\left[\left(\mathrm{UO}_{2}\right)\left(\mathrm{NO}_{3}^{-}\right)_{3}\right]^{-}$. This gas-phase ion is identical to the solution-based uranyl trinitrate complex, $\left[\left(\mathrm{UO}_{2}\right)^{2+}\left(\mathrm{NO}_{3}^{+}\right)_{3}\right]^{-}$, known to form in ketones, ethers and alcoholic solvents; the stability of the complex decreases in the order ketone $>$ ether $>$ alcohol $>$ water, which indicates a competition between solvent and nitrate for positions in the coordination sphere of $\mathrm{UO}_{2}^{2+}$ in solution [44]. Therefore, complexation by nitrates can easily occur during desolvation, when coordinating solvent molecules are stripped away. In the crystal structure of $\mathrm{Rb}\left[\left(\mathrm{UO}_{2}\right)\left(\mathrm{NO}_{3}\right)_{3}\right]$, each nitrate group chelates uranyl in a bidentate fashion, and the conformation around $\mathrm{U}(\mathrm{VI})$ is hexagonal bipyramid [45]. The coordination of $\left[\left(\mathrm{UO}_{2}\right)\left(\mathrm{NO}_{3}\right)_{3}\right]^{-}$in the gasphase is presumed to be similar.

The ion at $\mathrm{m} / \mathrm{z} 410$ can be assigned to $\left[\left(\mathrm{UO}_{2}\right)(\mathrm{O})\left(\mathrm{NO}_{3}\right)_{2}\right]^{-}$, which is supported by the observation that $\mathrm{O}^{2-}$ may undergo oxidation to form $\mathrm{O}^{-}$in gas-phase metal oxo-complexes [43]. However, note again that based solely on $\mathrm{m} / \mathrm{z}$ determination, the charge states of individual components of these complexes cannot be definitively known. The ion at $\mathrm{m} / \mathrm{z}$ 348 is assigned as $\left[\left(\mathrm{UO}_{2}\right)(\mathrm{O})\left(\mathrm{NO}_{3}\right)\right]^{-}$. Two ions of lower intensity appear at $\mathrm{m} / \mathrm{z} 366$ and $\mathrm{m} / \mathrm{z} 507$. The first may be assigned to $\left[\left(\mathrm{UO}_{2}\right)(\mathrm{OH})_{2}\left(\mathrm{NO}_{3}\right)\right]^{-}$, while the second corresponds to $\left[\left(\mathrm{UO}_{2}\right)\left(\mathrm{NO}_{3}\right)_{2}\left(\mathrm{CF}_{3} \mathrm{COO}\right)\right]^{-}$ and is the result of reaction with residual trifluoroacetate contaminant in the ESI source.

The corresponding doubly, triply, and quadruplycharged ions of the singly-charged species described above are seen in the spectrum below $\mathrm{m} / \mathrm{z} 250$ (Figure $4 \mathrm{~b}$, Table 3). If they are not exclusively overtones, these ions may contain uranium in the $\mathrm{U}(\mathrm{VI}), \mathrm{U}(\mathrm{V})$, or $\mathrm{U}(\mathrm{IV})$ oxidation states as a result of reduction reactions occurring during electrospray ionization in the negative ion mode. The appearance of the ions $\left[\left(\mathrm{UO}_{2}\right)(\mathrm{O})\left(\mathrm{NO}_{3}\right)_{2}\right]^{4-}(\mathrm{m} / \mathrm{z}$ 102.5), $\left[\left(\mathrm{UO}_{2}\right)(\mathrm{O})\left(\mathrm{NO}_{3}\right)\right]^{3-}(\mathrm{m} / \mathrm{z} 116)$, and $\left[\left(\mathrm{UO}_{2}\right)\left(\mathrm{NO}_{3}\right)_{3}\right]^{3-}$ $(\mathrm{m} / \mathrm{z} 152)$ is particularly interesting as they may contain uranium in the $\mathrm{U}(\mathrm{IV})$ oxidation state and indicate that both one and two electron additions to $\mathrm{UO}_{2}^{2+}$ may occur during electrospray ionization.

Product-ion spectra of the uranyl-nitrate complexes 
Table 3. Assignments for negative ions resulting from a solution containing $50 \mu \mathrm{M} \mathrm{UO}_{2}^{2+}$ and $100 \mu \mathrm{M} \mathrm{NO}_{3}{ }^{-}$in $95 \% \mathrm{CH}_{3} \mathrm{OH} / 5 \%$ $\mathrm{H}_{2} \mathrm{O}$ (vol/vol)

\begin{tabular}{|c|c|c|c|}
\hline Nominal $m / z^{a}$ & Observed $\mathrm{m} / \mathrm{z}$ & Calculated $\mathrm{m} / \mathrm{z}$ & Assignment \\
\hline 102.5 & 102.5040 & 102.5028 & {$\left[\left(\mathrm{UO}_{2}\right)(\mathrm{O})\left(\mathrm{NO}_{3}\right)_{2}\right]^{4-}$} \\
\hline 116 & 116.0081 & 116.0078 & {$\left[\left(\mathrm{UO}_{2}\right)(\mathrm{O})\left(\mathrm{NO}_{3}\right)\right]^{3-}$} \\
\hline 136.6 & 136.6734 & 136.6704 & {$\left[\left(\mathrm{UO}_{2}\right)(\mathrm{O})\left(\mathrm{NO}_{3}\right)_{2}\right]^{3-}$} \\
\hline 152 & 152.0017 & 152.0014 & {$\left[\left(\mathrm{UO}_{2}\right)\left(\mathrm{NO}_{3}\right)_{3}\right]^{3-}$} \\
\hline 174 & 174.0105 & 174.0117 & {$\left[\left(\mathrm{UO}_{2}\right)(\mathrm{O})\left(\mathrm{NO}_{3}\right)\right]^{2-}$} \\
\hline 183 & 183.0163 & 183.0169 & {$\left[\left(\mathrm{UO}_{2}\right)(\mathrm{OH})_{2}\left(\mathrm{NO}_{3}\right)\right]^{2-}$} \\
\hline $205^{*}$ & 205.0056 & 205.0056 & {$\left[\left(\mathrm{UO}_{2}\right)(\mathrm{O})\left(\mathrm{NO}_{3}\right)_{2}\right]^{2-}$} \\
\hline 228 & 228.0004 & 228.0020 & {$\left[\left(\mathrm{UO}_{2}\right)\left(\mathrm{NO}_{3}\right)_{3}\right]^{2-}$} \\
\hline 255 & 255.2316 & & \\
\hline 265 & 265.1469 & & \\
\hline 283 & 283.2633 & & \\
\hline 293 & 293.1783 & & \\
\hline 302 & 302.0301 & 302.0304 & {$\left[\left(\mathrm{UO}_{2}\right)(\mathrm{O})_{2}\right]^{-}$} \\
\hline 303 & 303.0379 & 303.0383 & {$\left[\left(\mathrm{UO}_{2}\right)(\mathrm{O})(\mathrm{OH})\right]^{-}$} \\
\hline 304 & 304.0445 & 304.0461 & {$\left[\left(\mathrm{UO}_{2}\right)(\mathrm{OH})_{2}\right]^{-}$} \\
\hline 317 & 317.0538 & 317.0539 & {$\left[\left(\mathrm{UO}_{2}\right)(\mathrm{O})\left(\mathrm{CH}_{3} \mathrm{O}\right)\right]^{-}$} \\
\hline 321 & 321.0043 & & \\
\hline 348 & 348.0217 & 348.0233 & {$\left[\left(\mathrm{UO}_{2}\right)(\mathrm{O})\left(\mathrm{NO}_{3}\right)\right]^{-}$} \\
\hline 364 & 364.0185 & 364.0183 & {$\left[\left(\mathrm{UO}_{2}\right)(\mathrm{O})_{2}\left(\mathrm{NO}_{3}\right)\right]^{-}$} \\
\hline 366 & 366.0340 & 366.0339 & {$\left[\left(\mathrm{UO}_{2}\right)(\mathrm{OH})_{2}\left(\mathrm{NO}_{3}\right)\right]^{-}$} \\
\hline 367 & 367.0222 & & \\
\hline 367 & 367.0401 & 367.0417 & {$\left[\left(\mathrm{UO}_{2}\right)(\mathrm{OH})_{2}\left(\mathrm{NO}_{3}\right)\left(\mathrm{H}_{2} \mathrm{O}\right)\right]^{-}$} \\
\hline 368 & 368.0468 & 368.0465 & {$\left[\left(\mathrm{UO}_{2}\right)(\mathrm{OD})_{2}\left(\mathrm{NO}_{3}\right)\right]^{-}$} \\
\hline 368 & & & {$\left[\left(\mathrm{UO}_{2}\right)(\mathrm{O})\left(\mathrm{NO}_{3}\right)\left(\mathrm{D}_{2} \mathrm{O}\right)\right]^{-}$} \\
\hline 382 & 382.9930 & & contaminant \\
\hline 394 & 394.0178 & & \\
\hline $410^{*}$ & 410.0112 & 410.0112 & {$\left[\left(\mathrm{UO}_{2}\right)(\mathrm{O})\left(\mathrm{NO}_{3}\right)_{2}\right]^{-}$} \\
\hline 411 & 411.0202 & & \\
\hline 412 & 412.0153 & & \\
\hline 413 & 413.0150 & 413.0147 & {$\left[\left(\mathrm{UO}_{2}\right)(\mathrm{F})\left(\mathrm{NO}_{3}\right)_{2}\right]^{-}$} \\
\hline 425 & 425.0361 & & \\
\hline 428 & 428.9866 & & \\
\hline 453 & 453.0317 & & \\
\hline 456 & 456.0022 & 456.0041 & {$\left[\left(\mathrm{UO}_{2}\right)\left(\mathrm{NO}_{3}\right)_{3}\right]^{-}$} \\
\hline 507 & 507.0042 & 507.0013 & {$\left[\left(\mathrm{UO}_{2}\right)\left(\mathrm{NO}_{3}\right)_{2}\left(\mathrm{CF}_{3} \mathrm{COO}\right)\right]^{-}$} \\
\hline
\end{tabular}

${ }^{a} \mathrm{~m} / \mathrm{z}$ Species denoted with * indicate ions used for calibration.

obtained using SORI-CID are shown in Figure 5a, b, c, and d. These fragmentation reactions follow a pattern similar to those described for transition-metal nitrate complexes, involving loss of $\mathrm{NO}_{2}$ and the formation of an oxo-nitrate complex followed by loss of $\mathrm{NO}_{3}$ and reduction of the metal [43]:

$$
\begin{aligned}
& {\left[\left(\mathrm{UO}_{2}^{2+}\right)\left(\mathrm{NO}_{3}^{-}\right)_{3}\right]^{-}(m / z 456) \rightarrow} \\
& {\left[\left(\mathrm{UO}_{2}^{2+}\right)\left(\mathrm{O}^{-}\right)\left(\mathrm{NO}_{3}^{-}\right)_{2}\right]^{-}(m / z 410)+\mathrm{NO}_{2}} \\
& {\left[\left(\mathrm{UO}_{2}^{2+}\right)\left(\mathrm{O}^{-}\right)\left(\mathrm{NO}_{3}^{-}\right)_{2}\right]^{-}(m / z 410) \rightarrow} \\
& {\left[\left(\mathrm{UO}_{2}^{+}\right)\left(\mathrm{O}^{-}\right)\left(\mathrm{NO}_{3}^{-}\right)\right]^{-}(m / z 348)+\mathrm{NO}_{3}}
\end{aligned}
$$

\begin{tabular}{|c|c|c|c|}
\hline Nominal $m / z^{a b}$ & Observed $\mathrm{m} / \mathrm{z}$ & Calculated $\mathrm{m} / \mathrm{z}$ & Assignment \\
\hline 348 & 348.0231 & 348.0233 & {$\left[\left(\mathrm{UO}_{2}\right)(\mathrm{O})\left(\mathrm{NO}_{3}\right)\right]^{-}$} \\
\hline 368 & 368.0465 & 368.0465 & {$\left[\left(\mathrm{UO}_{2}\right)(\mathrm{OD})_{2}\left(\mathrm{NO}_{3}\right)\right]^{-}$} \\
\hline 410 & 410.0111 & 410.0112 & {$\left[\left(\mathrm{UO}_{2}\right)(\mathrm{O})\left(\mathrm{NO}_{3}\right)_{2}\right]^{-}$} \\
\hline $456^{*}$ & 456.0041 & 456.0041 & {$\left[\left(\mathrm{UO}_{2}\right)\left(\mathrm{NO}_{3}\right)_{3}\right]^{-}$} \\
\hline 507 & 507.0015 & 507.0013 & {$\left[\left(\mathrm{UO}_{2}\right)\left(\mathrm{NO}_{3}\right)_{2}\left(\mathrm{CF}_{3} \mathrm{COO}\right)\right]^{-}$} \\
\hline 366 & 366.0326 & 366.0339 & {$\left[\left(\mathrm{UO}_{2}\right)(\mathrm{OH})_{2}\left(\mathrm{NO}_{3}\right)\right]^{-}$} \\
\hline 367 & 367.0220 & & \\
\hline 367 & 367.0393 & 367.0388 & {$\left[\left(\mathrm{UO}_{2}\right)(\mathrm{OH})(\mathrm{OD})\left(\mathrm{NO}_{3}\right)\right]^{-}$} \\
\hline $368 *$ & 368.0465 & 368.0465 & {$\left[\left(\mathrm{UO}_{2}\right)(\mathrm{OD})_{2}\left(\mathrm{NO}_{3}\right)\right]^{-}$} \\
\hline 384 & 384.0750 & 384.0747 & {$\left[\left(\mathrm{UO}_{2}\right)(\mathrm{O})\left(\mathrm{NO}_{3}\right)\left(\mathrm{CD}_{3} \mathrm{OD}\right)\right]^{-}$} \\
\hline 410 & 410.0117 & 410.0112 & {$\left[\left(\mathrm{UO}_{2}\right)(\mathrm{O})\left(\mathrm{NO}_{3}\right)_{2}\right]^{-}$} \\
\hline 456 & 456.0056 & 456.0041 & {$\left[\left(\mathrm{UO}_{2}\right)\left(\mathrm{NO}_{3}\right)_{3}\right]^{-}$} \\
\hline 348 & Not obs. & 348.0233 & {$\left[\left(\mathrm{UO}_{2}\right)(\mathrm{O})\left(\mathrm{NO}_{3}\right)\right]^{-}$} \\
\hline 367 & 367.0411 & 367.0388 & {$\left[\left(\mathrm{UO}_{2}\right)(\mathrm{OH})(\mathrm{OD})\left(\mathrm{NO}_{3}\right)\right]^{-}$} \\
\hline $368 *$ & 368.0465 & 368.0465 & {$\left[\left(\mathrm{UO}_{2}\right)(\mathrm{O})\left(\mathrm{NO}_{3}\right)\left(\mathrm{D}_{2} \mathrm{O}\right)\right]^{-}$} \\
\hline 384 & 384.0763 & 384.0747 & {$\left[\left(\mathrm{UO}_{2}\right)(\mathrm{O})\left(\mathrm{NO}_{3}\right)\left(\mathrm{CD}_{3} \mathrm{OD}\right)\right]^{-}$} \\
\hline
\end{tabular}

Table 4. Assignments for ions resulting from the reaction of $\left[\left(\mathrm{UO}_{2}\right)\left(\mathrm{NO}_{3}\right)_{2}\left(\mathrm{CF}_{3} \mathrm{COO}\right)\right]^{-},\left[\left(\mathrm{UO}_{2}\right)(\mathrm{OH})_{2}\left(\mathrm{NO}_{3}\right)\right]^{-}$and $\left[\left(\mathrm{UO}_{2}\right)(\mathrm{O})\left(\mathrm{NO}_{3}\right)\right]^{-}$ with $\mathrm{D}_{2} \mathrm{O}$

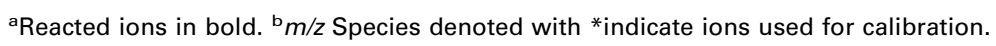




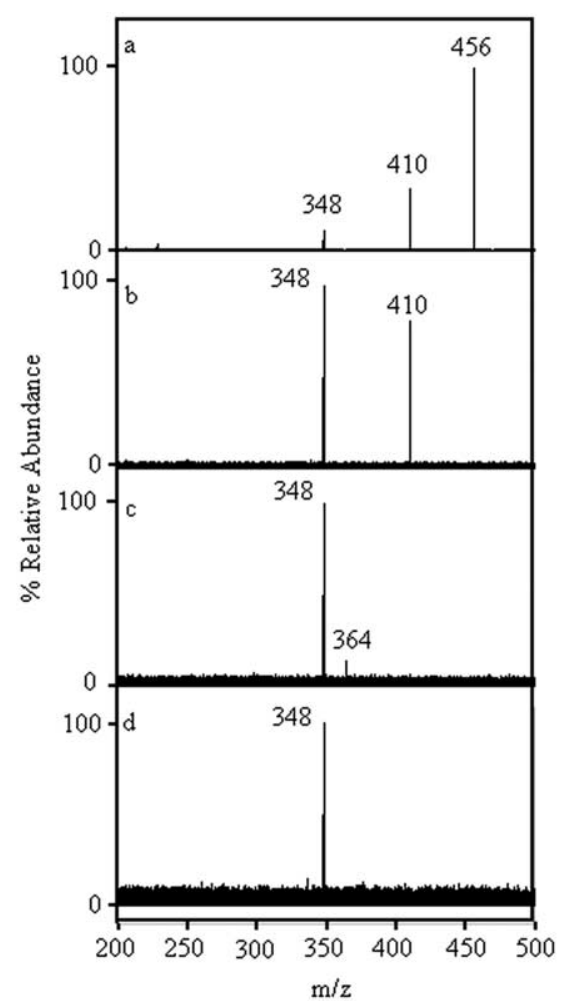

Figure 5. SORI-CID spectra of negatively charged ions at (a) $\mathrm{m} / \mathrm{z}$ 456, (b) $m / z$ 410, (c) $m / z 366$, and (d) $m / z 348$.

Note that tentative charge states of individual species of the complexes are suggested in eqs 1 and 2 to illustrate possible reduction of the uranyl center. The stable $\left[\left(\mathrm{UO}_{2}\right)(\mathrm{O})\left(\mathrm{NO}_{3}\right)\right]^{-}$ion $(\mathrm{m} / \mathrm{z} 348)$ cannot be further fragmented under our SORI-CID conditions. SORI-CID of $\left[\left(\mathrm{UO}_{2}\right)(\mathrm{OH})_{2}\left(\mathrm{NO}_{3}\right)\right]^{-}(\mathrm{m} / \mathrm{z} 366)$ results in a simple loss of $\mathrm{H}_{2} \mathrm{O}$, forming $\left[\left(\mathrm{UO}_{2}\right)(\mathrm{O})\left(\mathrm{NO}_{3}\right)\right]^{-}$at $\mathrm{m} / \mathrm{z} 348$. A small peak at $m / z 364$ is attributed to the loss of two hydrogen atoms or $\mathrm{H}_{2}$ from $\left[\left(\mathrm{UO}_{2}\right)(\mathrm{OH})_{2}\left(\mathrm{NO}_{3}\right)\right]^{-}$, with the formation of $\left[\left(\mathrm{UO}_{2}\right)(\mathrm{O})_{2}\left(\mathrm{NO}_{3}\right)\right]^{-}$(Figure $5 \mathrm{c}$ ).

\section{Ion-Molecule Reactions with Negative Ions Using $\mathrm{D}_{2} \mathrm{O}$}

$\mathrm{D}_{2} \mathrm{O}$ was used as a neutral reaction partner to compare the reaction chemistry of the anionic uranyl nitrate ions with the chemistry of the positive species discussed above. Representative spectra of the reaction region for the major ions in Figure 4a are collected in Figure 6a, b, c, and d. Assignments and exact mass values are provided in Table 4. All spectra shown in Figure $6 \mathrm{a}, \mathrm{b}, \mathrm{c}$, and $\mathrm{d}$ were obtained through selection and reaction of the ion of interest with $\mathrm{D}_{2} \mathrm{O}$ for $180 \mathrm{~s}$ at $2.4 \times 10^{-8}$ torr.

Examination of the spectra in Figure 6a, b, c, and d indicates that the negative uranyl nitrate-containing ions do not undergo the extensive series of reactions upon collision with $\mathrm{D}_{2} \mathrm{O}$ as seen for the positively charged uranyl nitrate species (Figure 2a, b, c, d, and Figure 3). With the exception of $\left[\left(\mathrm{UO}_{2}\right)(\mathrm{O})\left(\mathrm{NO}_{3}\right)\right]^{-}$at $\mathrm{m} / z 348, \mathrm{D}_{2} \mathrm{O}$ additions are not observed, indicating that these ions are coordinatively saturated.

Neither $\left[\left(\mathrm{UO}_{2}\right)\left(\mathrm{NO}_{3}\right)_{3}\right]^{-}\left(\mathrm{m} / \mathrm{z}\right.$ 456) nor $\left[\left(\mathrm{UO}_{2}\right)(\mathrm{O})\right.$ $\left.\left(\mathrm{NO}_{3}\right)_{2}\right]^{-}(m / z 410)$ react with $\mathrm{D}_{2} \mathrm{O}$ at the pressure used for these experiments (Figure $6 a$ and $b$ ). The $\left[\left(\mathrm{UO}_{2}\right)\left(\mathrm{NO}_{3}\right)_{3}\right]^{-}$ion, with its presumed bidentate nitrate groups, is coordinatively saturated and cannot accept a $\mathrm{D}_{2} \mathrm{O}$ ligand. $\left[\left(\mathrm{UO}_{2}\right)(\mathrm{O})\left(\mathrm{NO}_{3}\right)_{2}\right]^{-}$most likely has a pentagonal bipyramidal conformation with two bidentate nitrate groups and a single oxygen ligand. Gas-phase uranyl-containing ions with this type of conformation are quite stable [19]. Assuming bidentate coordination by nitrate, $\left[\left(\mathrm{UO}_{2}\right)(\mathrm{OH})_{2}\left(\mathrm{NO}_{3}\right)\right]^{-}(\mathrm{m} / \mathrm{z} 366)$ presumably has a conformation in which there is coordination at only four equatorial sites, yet the complex does not accept a $\mathrm{D}_{2} \mathrm{O}$ ligand but only undergoes an $\mathrm{H} / \mathrm{D}$ exchange reaction to form $\left[\left(\mathrm{UO}_{2}\right)(\mathrm{OD})_{2}\left(\mathrm{NO}_{3}\right)\right]^{-}(\mathrm{m} / \mathrm{z}$ 368). Only $\left[\left(\mathrm{UO}_{2}\right)(\mathrm{O})\left(\mathrm{NO}_{3}\right)\right]^{-}\left(\mathrm{m} / z\right.$ 348) can accept a $\mathrm{D}_{2} \mathrm{O}$ ligand, forming $\left[\left(\mathrm{UO}_{2}\right)(\mathrm{O})\left(\mathrm{NO}_{3}\right)\left(\mathrm{D}_{2} \mathrm{O}\right)\right]^{-}$at $\mathrm{m} / z$ 368. In both of the latter two cases, a small ion at $\mathrm{m} / \mathrm{z} 384$ appears as a reaction product. This ion is tentatively assigned to $\left[\left(\mathrm{UO}_{2}\right)(\mathrm{O})\left(\mathrm{NO}_{3}\right)\left(\mathrm{CD}_{3} \mathrm{OD}\right)\right]^{-}$and may result from trace amounts of $\mathrm{CD}_{3} \mathrm{OD}$ in the $\mathrm{D}_{2} \mathrm{O}$ reagent used for these reactions. Finally, we note that the ions at $\mathrm{m} / \mathrm{z}$ 410, $\left[\left(\mathrm{UO}_{2}\right)(\mathrm{O})\left(\mathrm{NO}_{3}\right)_{2}\right]^{-}$and 456, $\left[\left(\mathrm{UO}_{2}\right)\left(\mathrm{NO}_{3}\right)_{3}\right]^{-}$, in Figure $6 \mathrm{c}$, are presumably the result of multiple collision reactions occurring with nitrate-containing species in the FT-ICR cell.

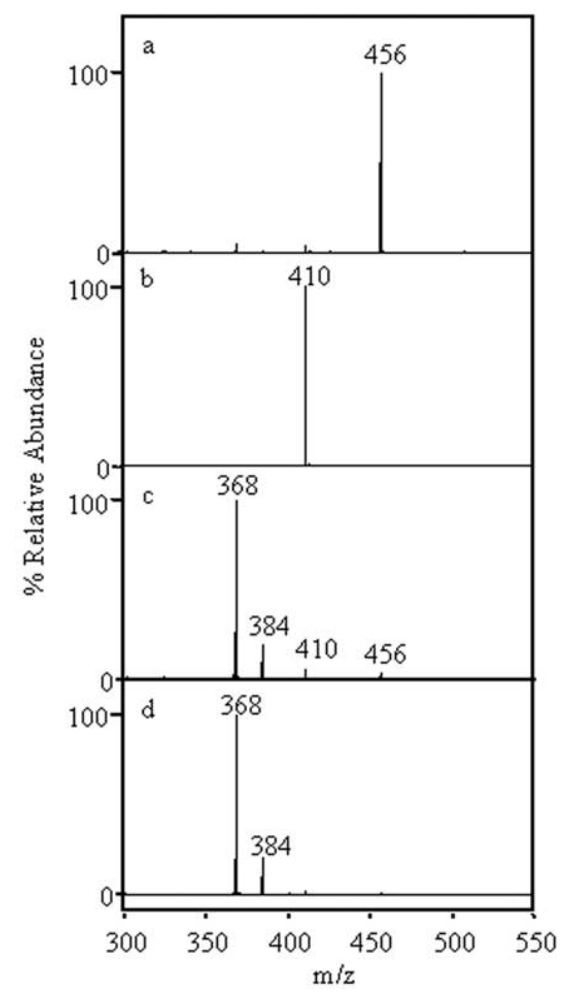

Figure 6. Ions produced by reactions (180 s reaction time) between $\mathrm{D}_{2} \mathrm{O}\left(2.4 \times 10^{-8}\right.$ torr $)$ and negative ions at (a) $\mathrm{m} / \mathrm{z} 456$, (b) $\mathrm{m} / \mathrm{z} 410$, (c) $\mathrm{m} / \mathrm{z} 366$, and (d) $\mathrm{m} / \mathrm{z} 348$. 


\section{Conclusions}

Electrospray ionization of uranyl nitrate solutions generates a wide variety of positively and negatively charged ions, including complex adducts of uranyl, methoxy, hydroxy, and nitrate ligands, as well as oxygen. In the positive ion mode, the ions detected are highly sensitive to instrumental tuning parameters such as quadrupole operating frequency and trapping time. Ions formed are of varying stability and reactivity with some undergoing extensive fragmentation upon collision with $\mathrm{D}_{2} \mathrm{O}$ and others not. The extent of coordinative unsaturation of both positive and negative ions can be investigated using $\mathrm{D}_{2} \mathrm{O}$ and $\mathrm{CD}_{3} \mathrm{OD}$ as neutral reaction partners within the applied pressure range. Electron-transfer reactions, presumably occurring during electrospray, can result in reduction of $U(V I)$ to U(V) and perhaps even U(IV). SORI-CID fragmentation processes are often coupled with electron-transfer involving both the uranyl moiety and the oxygen ligand, if present.

This simple uranyl nitrate system provides a wealth of both simple and complex ions and illustrates the complexity of the chemical reactions that occur between injection of solution into the ESI source region and analysis in the ICR cell. Most, if not all, of the ions detected were not solution-phase species, but were generated during desolvation or during gas-phase reactions in the instrument. This work demonstrates the utility of FT-ICR, with its ultrahigh resolution and mass accuracy, for the study of the gas-phase reaction chemistry of uranyl and its complexes.

\section{Acknowledgments}

The authors gratefully acknowledge support of this research by the National Institutes of Health through the Native American Cancer Research Partnership funded through the Minority Institute Cancer Control Program of the National Cancer Institute (U54CA96281).

\section{References}

1. Wu, Q; Cheng, X: Hofstadler, S. A. Smith, R. D. Specific MetalOligonucleotide Binding Studied by High Resolution Tandem Mass Spectrometry. J. Mass Spectrom. 1996, 31, 669-675.

2. Moulin, C.; Charron, N.; Plancque, G.; Virelizier, H. Speciation of Uranium by Electrospray Ionization Mass Spectrometry: Comparison with Time-Resolved Laser-Induced Fluorescence. Appl. Spectrosc. 2000, 54, 843-848.

3. Jacopin, C.; Sawicki, M.; Plancque, G.; Doizi, D.; Taran, F.; Ansoborlo, E.; Amekraz, B.; Moulin, C. Investigation of the Interaction Between 1-Hydroxyethane-1,1-Diphosphonic Acid (HEDP) and Uranium(VI). Inorg. Chem. 2003, 42, 5015-5022.

4. Pasilis, S. P.; Pemberton, J. E. Speciation and Coordination Chemistry of Uranyl(VI)-Citrate Complexes in Aqueous Solution. Inorg. Chem. 2003, 42, 6793-6800.

5. Groenewold, G. S.; Van Stipdonk, M. J.; Gresham, G. L.; Chien, W.; Bulleigh, K.; Howard, A. Collision-Induced Dissociation Tandem Mass Spectrometry of Desferrioxamine Siderophore Complexes from Electrospray Ionization of $\mathrm{UO}_{2}^{2+}, \mathrm{Fe}^{3+}$, and $\mathrm{Ca}^{2+}$ Solutions. J. Mass Spectrom. 2004, 39, 752-761.

6. Heinemann, C.; Cornehl, H. H.; Schwarz, H. Hydrocarbon Activation by "Bare" Uranium Cations: Formation of a Cationic Uranium-Benzene Complex from Three Ethylene Units. J. Organomet. Chem. 1995, 501, 201-209.

7. Gibson, J. K. Gas-Phase f-Element Organometallic Chemistry: Reactions of Cyclic Hydrocarbons with $\mathrm{Th}^{+}, \mathrm{U}^{+}, \mathrm{ThO}^{+}, \mathrm{UO}^{+}$, and Lanthanide Ions, $\mathrm{Ln}^{+}$. Organometallics 1997, 16, 4214-4222.
8. Gibson, J. K. Gas-Phase Transuranium Organometallic Chemistry: Reactions of $\mathrm{Np}^{+}, \mathrm{Pu}^{+}, \mathrm{NpO}^{+}$, and $\mathrm{PuO}^{+}$with Alkenes. J. Am. Chem. Soc. 1998, 120, 2633-2640.

9. Gibson, J. K. Gas-Phase Transuranium Chemistry: Reactions of Actinide Ions with Alcohols and Thiols. J. Mass Spectrom. 1999, 34, 1166-1177.

10. Marcalo, J.; Leal, J. P.; Pires de Matos, A. Gas-Phase Actinide Ion Chemistry: FT-ICR/MS Study of the Reactions of Thorium and Uranium Metal and Oxide Ions with Arenes. Organometallics 1997, 16, 4581-4588.

11. Armentrout, P. B.; Hodges, R. V.; Beauchamp, J. L. Endothermic Reactions of Uranium Ions with $\mathrm{N}_{2}, \mathrm{D}_{2}$, and $\mathrm{CD}_{4}$. J. Chem. Phys. 1977, 66, 4683-4688.

12. Armentrout, P. B.; Beauchamp, J. L. Reactions of $\mathrm{U}^{+}$and $\mathrm{UO}^{+}$with $\mathrm{O}_{2}$, $\mathrm{CO}, \mathrm{CO}_{2}, \mathrm{COS}, \mathrm{CS}_{2}$, and $\mathrm{D}_{2} \mathrm{O}$. Chem. Phys. 1980, 50, 27-36.

13. Cornehl, H. H.; Heinemann, C.; Marcalo, J.; Pires de Matos, A.; Schwarz, $\mathrm{H}$. The "Bare" Uranyl(2+) Ion, $\mathrm{UO}_{2}^{2+}$. Angew. Chem. Int. Ed. Engl. 1996, 35, 891-894.

14. Jackson, G. P.; King, F. L.; Goeringer, D. E.; Duckworth, D. C. Gas-Phase Reactions of $\mathrm{U}^{+}$and $\mathrm{U}^{2+}$ with $\mathrm{O}_{2}$ and $\mathrm{H}_{2} \mathrm{O}$ in a Quadrupole Ion Trap. J. Phys. Chem. A 2002, 106, 7788-7794.

15. Jackson, G. P.; Gibson, J. K.; Duckworth, D. C. Gas-Phase Reactions of Bare and Ligated Uranium Ions with Sulfur Hexafluoride. J. Phys. Chem. A 2004, 108, 1042-1051.

16. Gibson, J. K.; Haire, R. G.; Santos, M.; Marcalo, J.; Pires de Matos, A. Oxidation Studies of Dipositive Actinide Ions, $\mathrm{An}^{2+}(\mathrm{An}=\mathrm{Th}, \mathrm{U}$, $\mathrm{Np}, \mathrm{Pu}, \mathrm{Am})$ in the Gas Phase: Synthesis and Characterization of the Isolated Uranyl, Neptunyl, and Plutonyl Ions $\mathrm{UO}_{2}^{2+}(\mathrm{g}), \mathrm{NpO}_{2}^{2+}(\mathrm{g})$, and $\mathrm{PuO}_{2}^{2+}(\mathrm{g})$. J. Phys. Chem. A 2005, 109, 2768-2781.

17. Gresham, G. L.; Gianotto, A. K.; de Harrington, P.; Cao, L.; Scott, J. R.; Olson, J. E.; Appelhans, A. D.; Van Stipdonk, M. J.; Groenewold, G. S. Gas-Phase Hydration of U(IV), U(V), and U(VI) Dioxo Monocations. J. Phys. Chem. A 2003, 107, 8530-8538.

18. Van Stipdonk, M.; Anbalagan, V.; Chien, W.; Gresham, G.; Groenewold, G.; Hanna, D. Elucidation of the Collision Induced Dissociation Pathways of Water and Alcohol Coordinated Complexes Containing the Uranyl Ion. J. Am. Soc. Mass Spectrom. 2003, 14, 1205-1214.

19. Chien, W. Anbalagan, V.: Zandler, M.: Van Stipdonk, M. J. Intrinsic Hydration of Monopositive Uranyl Hydroxide, Nitrate, and Acetate Cations. J. Am. Soc. Mass Spectrom. 2004, 15, 777-783.

20. Van Stipdonk, M. J.; Chien, W.; Anbalagan, V.; Gresham, G.; Groenewold, G. Oxidation of 2-Propanol Ligands during Collision-Induced Dissociation of a Gas-Phase Uranyl Complex. Int. J. Mass Spectrom. 2004, 237, 175-183

21. Van Stipdonk, M. J.; Chien, W.; Anbalagan, V.; Bulleigh, K.; Hanna, D.; Groenewold, G. Gas-Phase Complexes Containing the Uranyl Ion and Acetone. J. Phys. Chem. A 2004, 108, 10448-10457.

22. Jiao, C. Q.; Ranatunga, D. R. A.; Vaugn, W. E.; Freiser, B. S. A Pulsed-Leak Valve for Use with Ion Trapping Mass Spectrometers. J. Am. Soc. Mass Spectrom. 1996, 7, 118-122.

23. Campbell, S.; Rodgers, M. T.; Marzluff, E. M.; Beauchamp, J. L. Deuterium Exchange Reactions as a Probe of Biomolecule Structure. Fundamental Studies of Gas Phase H/D Exchange Reactions of Protonated Glycine Oligomers with $\mathrm{D}_{2} \mathrm{O}, \mathrm{CD}_{3} \mathrm{OD}, \mathrm{CD}_{3} \mathrm{CO}_{2} \mathrm{D}$, and $\mathrm{ND}_{3}$. J. Am. Chem. Soc. 1995, 117, 12840-12854.

24. Bartmess, J. E.; Georgiadis, R. M. Empirical Methods for Determination of Ionization Gauge Relative Sensitivities for Different Gases. Vacuum 1983, 33, 149-153.

25. Anderson, U. N.; Bojesen, G. Gas-Phase Ion/Molecule Reactions of Doubly Charged Clusters of $\mathrm{Fe}^{2+}$ or $\mathrm{Mn}^{2+}$ and Methanol with Ammonia. Int. J. Mass Spectrom. Ion Processes 1996, 153, 1-7.

26. Reid, G. E.; O'Hair, R. A. J.; Styles, M. L.; McFayden, W. D.; Simpson, R. J. Gas Phase Ion-Molecule Reactions in a Modified Ion Trap: H/D Exchange of Noncovalent Complexes and Coordinatively Unsaturated Platinum Complexes. Rapid Commun. Mass Spectrom. 1998, 12, 17011708.

27. Nielsen, S. B.; Bojesen, G. Ligand and $\mathrm{H} / \mathrm{D}$ Exchange of $\mathrm{Mn}\left(\mathrm{H}_{2} \mathrm{O}\right)_{6}^{2+}$ and $\mathrm{Cu}(\mathrm{OH})\left(\mathrm{H}_{2} \mathrm{O}\right)_{4}^{+}$in the Gas Phase. Chem. Commun. 1998, 613-614.

28. Molina-Svendsen, H.; Bojesen, G.; McKenzie, C. J. Gas-Phase Reactivity of Coordinatively Unsaturated Transition Metal Complex Ions Toward Molecular Oxygen. Inorg. Chem. 1998, 37, 1981-1983.

29. Vachet, R. W.; Hartman, J. A. R.; Callahan, J. H. Ion-Molecule Reactions in a Quadrupole Ion Trap as a Probe of the Gas-Phase Structure of Metal Complexes. J. Mass Spectrom. 1998, 33, 1209-1225.

30. Vachet, R. W.; Callahan, J. H. Quadrupole Ion Trap Studies of the Structure and Reactivity of Transition Metal Ion Pair Complexes. J. Mass Spectrom. 2000, 35, 311-320.

31. Vachet, R. W.; Hartman, J. R.; Gertner, J. W.; Callahan, J. H. Investigation of Metal Complex Coordination Structure using Collision-Induced Dissociation and Ion-Molecule Reactions in a Quadrupole Ion Trap Mass Spectrometer. Int. J. Mass Spectrom. 2001, 204, 101-112.

32. Combariza, M. Y. Gas-Phase Ion-Molecule Reactions of Transition Metal Complexes: The Effect of Different Coordination Spheres on Complex Reactivity. J. Am. Soc. Mass Spectrom. 2002, 13, 813-825.

33. Combariza, M. Y.; Vachet, R. W. The Utility of Ion-Molecule Reactions in a Quadrupole Ion Trap Mass Spectrometer for Analyzing Metal Complex Coordination Structure. Anal. Chim. Acta 2003, 496, 233-248.

34. Combariza, M. Y.; Fermann, J. T.; Vachet, R. W. Are Gas-Phase Reactions of Five-Coordinate Divalent Metal Ion Complexes Affected by Coordination Geometry? Inorg. Chem. 2004, 43, 2745-2753. 
35. Jennings, K. R.; Kemp, T. J.; Read, P. A. Cluster Formation in the Fast Atom Bombardment (FAB) Mass Spectra of Dioxouranium(VI) Dinitrate and Diacetate. Inorg. Chim. Acta 1989, 157, 157-159.

36. Kemp, T. J.; Jennings, K. R.; Read, P. A. Formation and Decomposition of Uranium-Oxygen Clusters in Fast Atom Bombardment of Dioxouranium(VI) Salts. J. Chem. Soc. Dalton Trans. 1995, 1995, 885-889.

37. Pospieszna-Markiewicz, I.; Radecka-Paryzek, W. The New Macrocyclic and Acyclic Complexes of the Uranyl Ion: Uranyl-Oxygen Cluster Formation in Fast Atom Bombardment Mass Spectra. J. Alloys Compd. 2004, 374, 253-257.

38. Brown, D. A.; Ismail, S. Synthesis of Dioxouranium Dialkylhydromates: Cluster Formation in Fast Atom Bombardment Mass Spectra (FAB-MS). Inorg. Chim. Acta 1990, 171, 42-43.

39. Formosinho, S. J.; Burrows, H. D.; da Graca-Miguel, M.; Azenha, M. E. D. G.; Saraiva, I. M.; Ribeiro, A. C. D. N.; Khudyakov, I. V.; Gasanov, R. G.; Bolte, M.; Sarakha, M. Deactivation Processes of the Lowest Excited State of $\left[\mathrm{UO}_{2}\left(\mathrm{H}_{2} \mathrm{O}\right)_{5}\right]^{2+}$ in Aqueous Solution. Photochem. Photobiol. Sci. 2003, 2, 569-575.
40. Henis, J. M. S. An Ion Cyclotron Resonance Study of Ion-Molecule Reactions in Methanol. J. Am. Chem. Soc. 1968, 90, 844-851.

41. Kleingeld, J. C.; Nibbering, N. M. M. A Fourier Transform Ion Cyclotron Resonance Study of the Mechanism of Formation of Protonated Dimethyl Ether from Methanol by Use of Naturally Occurring ${ }^{18} \mathrm{O}$. Org. Mass Spectrom. 1982, 17, 136-139.

42. Mollah, S.; Pris, A. D.; Johnson, S. K.; Gwizdala, A. B.; Houk, R. S. Identification of Metal Cations, Metal Complexes, and Anions by Electrospray Mass Spectrometry in the Negative Ion Mode. Anal. Chem. 2000, 72, 985-991.

43. Li, F.; Byers, M. A.; Houk, R. S. Tandem Mass Spectrometry of Metal Nitrate Negative Ions Produced by Electrospray Ionization. J. Am. Soc. Mass Spectrom. 2003, 14, 671-679.

44. Kaplan, L.; Hildebrandt, R. A.; Ader, M. The Trinitrouranyl Ion in Organic Solvents. J. Inorg. Nucl. Chem. 1956, 2, 153-163.

45. Barclay, G. A.; Sabine, T. M.; Taylor, J. C. The Crystal Structure of Rubidium Uranyl Nitrate: A Neutron Diffraction Study. Acta Crystallogr. 1965, 19, 205-209. 